\section{RIPK3/Fas-Associated Death Domain Axis Regulates Pulmonary Immunopathology to Cryptococcal Infection Independent of Necroptosis}

\author{
Zhenzong $\mathrm{Fa}^{1,2,3 \dagger}$, Qun Xie ${ }^{4,5+}$, Wei Fang ${ }^{2,6 t}$, Haibing Zhang ${ }^{5}$, Haiwei Zhang ${ }^{5}$, Jintao $\mathrm{Xu}^{3}$, \\ Weihua Pan ${ }^{1,2}$, Jinhua $X^{6}{ }^{6}$, Michal A. Olszewski ${ }^{2,3 *}$, Xiaoming Deng ${ }^{4 \star}$ and Wanqing Liao ${ }^{1,2 *}$ \\ 'PLA Key Laboratory of Mycosis, Department of Dermatology and Venereology, Changzheng Hospital, Shanghai, China, \\ ${ }^{2}$ Shanghai Key Laboratory of Molecular Medical Mycology, Shanghai Institute of Medical Mycology, Second Military Medical \\ University, Shanghai, China, ${ }^{3}$ Division of Pulmonary and Critical Care Medicine, Department of Internal Medicine, University \\ of Michigan Health System, Ann Arbor, MI, United States, ${ }^{4}$ Department of Anesthesiology and Intensive Care, Changhai \\ Hospital, Second Military Medical University, Shanghai, China, ${ }^{5}$ Key Laboratory of Nutrition and Metabolism, Institute for \\ Nutritional Sciences, Shanghai Institutes for Biological Sciences, Chinese Academy of Sciences, Shanghai, China, \\ ${ }^{6}$ Department of Dermatology, Huashan Hospital, Fudan University, Shanghai, China
}

Fas-associated death domain (FADD) and receptor interacting protein kinase 3 (RIPK3) are multifunctional regulators of cell death and immune response. Using a mouse model of cryptococcal infection, the roles of FADD and RIPK3 in anti-cryptococcal defense were investigated. Deletion of RIPK3 alone led to increased inflammatory cytokine production in the Cryptococcus neoformans-infected lungs, but in combination with FADD deletion, it led to a robust Th1-biased response with M1-biased macrophage activation. Rather than being protective, these responses led to paradoxical $C$. neoformans expansion and

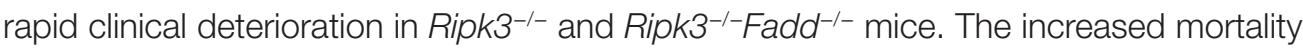

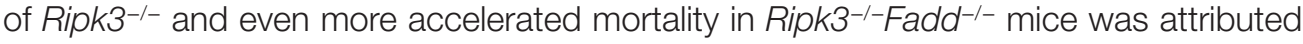
to profound pulmonary damage due to neutrophil-dominant infiltration with prominent upregulation of pro-inflammatory cytokines. This phenomenon was partially associated with selective alterations in the apoptotic frequency of some leukocyte subsets, such as eosinophils and neutrophils, in infected Ripk3 ${ }^{-/}$Fadd $^{-1-}$ mice. In conclusion, our study shows that RIPK3 in concert with FADD serve as physiological "brakes," preventing the development of excessive inflammation and Th1 bias, which in turn contributes to pulmonary damage and defective fungal clearance. This novel link between the protective effect of FADD and RIPK3 in antifungal defense and sustenance of immune homeostasis may be important for the development of novel immunomodulatory therapies against invasive fungal infections.

Keywords: Cryptococcus neoformans, immune responses, inflammation, Fas-associated death domain, receptorinteracting serine/threonine kinase 3

\section{INTRODUCTION}

Invasive fungal infections have become an increasingly significant challenge to public health due to the ever-increasing population of immunosuppressed patients, associated with aging of the global population, immunosuppressive infections such as HIV, and the growing use of immunosuppressive therapies. Among the major fungal pathogens, Cryptococcus neoformans causes life-threatening 
invasive infections in both immunocompromised and immunocompetent hosts $(1,2)$. As supported by evidence from both the clinic and animal infection models, an insufficiency of Th1 and Th17 responses and subsequent classical activation of macrophages are major triggers of cryptococcal infection. However, emerging evidence supports the view that the excessive inflammation and pathology is frequently derived by a Th1 response, which is often initiated during highly active antiretroviral therapy (HAART) in $\mathrm{HIV}^{+}$patients with cryptococcosis. This paradoxical response, known as immune reconstitution inflammatory syndrome (IRIS), contributes to worsening symptoms and patient mortality despite ongoing antifungal and HAART treatments (3). This unique clinical problem underscores the importance of immunoregulatory processes during opportunistic fungal infections of which many aspects remain to be elucidated.

Fas-associated death domain protein (FADD) is known as a critical mediator of death receptor-triggered extrinsic apoptosis, which plays a role in removing "no longer needed" inflammatory cells, thereby serving as a crucial immune-regulatory pathway at the site of infection, preventing excessive inflammation $(4,5)$. Besides its role in apoptosis, FADD also has been shown to function in regulating cell cycle progression $(6,7)$, cytokine signaling $(8,9)$, and T-cell activation (10), which are all involved in regulation of immune responses. Targeted deletion of FADD in mice causes embryonic lethality due to spontaneous activation of another programmed cell death (PCD) pathway, necroptosis (11). However, co-deletion of receptor interacting protein kinase 3 (RIPK3), which is an essential serine/threonine kinase for necroptosis, rescues these mice (12). In addition to necroptosis, RIPK3 has also been reported as an important inflammatory signal adaptor because it functions in NF- $\mathrm{KB}$ activation, inflammasome activation, and cytokine signaling (13-15) and participates in the pathogenesis of several inflammatory diseases. However, it remains unknown whether these molecules play important roles during immune responses to fungal infections.

Here, we explored the roles of FADD and RIPK3 in a mouse model of cryptococcal infection and identified previously unknown, critical contributions of these molecules in pulmonary immune responses to cryptococcal infection. Deletion of FADD and RIPK 3 induced robust Th1 responses, which, paradoxically, led to $C$. neoformans expansion and increased mortality in the infected mice. These effects were attributed to an excessive accumulation of neutrophils, over exuberant inflammatory cytokine production, and development of severe lung pathology. Collectively, these findings establish a novel link between these PCD components and immune response to cryptococcal challenge, demonstrating the crucial importance of FADD and RIPK3 in maintaining immune homeostasis during invasive fungal infection.

\section{MATERIALS AND METHODS}

\section{Mice}

Female wild-type (WT) C57BL/6 mice were housed in a specific pathogen-free facility. Ripk $3^{-1-}$ mice have been previ-

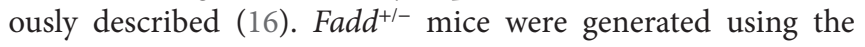

CRISPR-Cas9mutationsystem(ShanghaiBiorayLaboratory,Inc.). A 100-bp deletion was introduced into exon 1 of the Fadd gene (Figure S1 in Supplementary Material). Because ablation of Fadd in mice causes embryonic death, we crossed the Ripk $3^{-/-}$mice

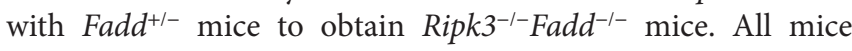
genotypes were confirmed by PCR (Figure S1 in Supplementary Material). Mice were 8-10 weeks old at the time of infection and were humanely euthanized by $\mathrm{CO}_{2}$ inhalation at the time of data collection. Animal experiments were conducted in accordance with the National Institutes of Health Guide for the Care and Use of Laboratory Animals with the approval of the Scientific Investigation Board of Second Military Medical University.

\section{Cryptococcus neoformans}

Encapsulated C. neoformans strain H99 (serotype A) was recovered from $10 \%$ glycerol-frozen stocks stored at $-80^{\circ} \mathrm{C}$. The strains were cultured on yeast extract-peptone-dextrose agar plates at $30^{\circ} \mathrm{C}$. Liquid cultures were grown in Sabouraud dextrose broth at $30^{\circ} \mathrm{C}$ for $20-24 \mathrm{~h}$ in a shaking incubator at $180 \mathrm{rpm}$. Fungal cells were centrifuged at $2,000 \times g$ for $3 \mathrm{~min}$, washed three times, and resuspended in sterile PBS.

\section{Inoculation}

Mice were anesthetized by intraperitoneal injection of ketamine (100 mg/kg, Sigma, St. Louis, MO, USA) and secured onto a clean foam board (17). Next, $50 \mu \mathrm{l}\left[10^{5}\right.$ colony-forming units (CFU)] of the washed yeast $\left(2 \times 10^{6}\right.$ yeast cells $/ \mathrm{ml}$ in sterile PBS) were used for intranasal infection as previously described (18). After inoculation, the mice were kept warm and monitored during recovery from anesthesia.

\section{Tissue Collection and Lung Leukocyte Isolation}

The procedures were performed as previously described with modifications (19). At the time of data collection, the mice were sacrificed and perfused with $5 \mathrm{ml}$ sterile PBS. The lungs were removed, minced with scissors, and added to homogenization gentleMACS $\mathrm{C}$ tubes containing proprietary catalysts for mechanical and enzymatic digestion (Miltenyi Biotec, Auburn, CA, USA). This process was followed by lung tissue homogenization using the gentle MACS dissociator (Miltenyi Biotec) and incubation at $37^{\circ} \mathrm{C}$ for $30 \mathrm{~min}$ in $4 \mathrm{ml} /$ mouse digestion buffer (RPMI 1640, 5\% fetal bovine serum, penicillin, and streptomycin, Invitrogen, Grand Island, NY, USA), $1 \mathrm{mg} / \mathrm{ml}$ collagenase A (Roche Diagnostics, Indianapolis, IN, USA), and $30 \mathrm{~g} / \mathrm{ml}$ DNase I (Sigma, St. Louis, MO, USA). Erythrocytes were removed using $1 \times$ RBC lysis buffer (eBioscience, San Diego, CA, USA). Homogenized tissue was then passed through a $70 \mu \mathrm{m}$ cell strainer (BD Falcon, Bedford, MA, USA) and centrifuged at $300 \times g$ for $10 \mathrm{~min}$ to pellet the cells. The filtrate was centrifuged for $25 \mathrm{~min}$ at $1,500 \times g$ in the presence of $20 \%$ Percoll (Sigma, St. Louis, MO, USA) in complete RPMI 1640 medium (RPMI $1640,5 \% \mathrm{FBS}$, penicillin, and streptomycin) with no brake to separate leukocytes from cell debris and epithelial cells. Leukocyte pellets were resuspended in $5 \mathrm{ml}$ complete RPMI 1640 medium 
and counted in a hemocytometer using trypan blue staining to exclude dead cells.

\section{Tissue CFU Assay}

For determining the fungal burden in the lungs, brains, and spleens, tissues were removed and homogenized in $1 \mathrm{ml}$ sterile PBS. Ten-fold dilutions of the samples were plated in duplicate on Sabouraud dextrose agar plates. Colonies were counted after $48 \mathrm{~h}$ of growth at $30^{\circ} \mathrm{C}$, and CFU were calculated on a per-gram basis.

\section{Cytokine Analysis}

Mouse serum was obtained from blood samples collected by heart puncture before lung excision and centrifugation at $10,000 \times g$ for $10 \mathrm{~min}$. Homogenates of lungs were centrifuged, and supernatants were diluted for cytokine analysis. Leukocytes isolated from mice lung were plated at $10^{7} \mathrm{cells} / \mathrm{ml}$, and supernatants were collected by centrifugation of the culture medium. Mouse TNF $\alpha, \operatorname{IFN} \gamma$, IL-1 $\alpha$, IL-1 $\beta$, IL-4, IL-6, IL-12, IL-17A, and IL-33 ELISA kits were from eBioscience. CXCL1 was from Raybio (Norcross, GA, USA).

\section{Flow Cytometry Analysis of Leukocyte Populations}

For the flow cytometry experiments, antibodies were purchased from eBioscience, BioLegend, or BD Biosciences, including anti-murine CD16/CD32; CD45 conjugated to PerCP-Cy5.5; CD3, CD193, CD80, IFN $\gamma$, and annexin V conjugated to FITC; CD4, Ly6G, and Siglec F conjugated to APC; CD8, CD11c, CD19, and CD80 conjugated to PE-Cy7; CD19, CD40, F4/80, and IL-4 conjugated to $\mathrm{PE}$; $\mathrm{CD} 11 \mathrm{~b}, \mathrm{CD} 326$, and $\mathrm{MHC}$ II conjugated to APC-Cy7; and CD19, CD80, and Ly6C conjugated to BV421.

Leukocytes were isolated from the lung and lymph nodes of mice. Cell surface immunofluorescence staining involved the addition of a fluorochrome-conjugated antibody mixture containing antibodies specific to various leukocyte subpopulations to the staining buffer. Cells were incubated on ice for $30 \mathrm{~min}$ in the dark and washed twice with PBS. For intracellular cytokine staining, cells were fixed in IC fixation buffer and stimulated with cell stimulation cocktail (plus protein transportation inhibitors) from eBioscience. Cells were resuspended in permeabilization buffer (eBioscience) and stained with intracellular antibody cocktail. After staining, cells were immediately analyzed by flow cytometry (FACSAria III, BD Biosciences). FlowJo (For Mac OS $\mathrm{X}$, version X 10.0.7r2, Tree Star, San Carlos, CA, USA) was used for data analysis. Leukocyte populations were identified using the following markers as previously described $(20,21)$ : neutrophils $\left(\mathrm{CD} 45^{+} \mathrm{Ly}_{6} \mathrm{G}^{+} \mathrm{CD} 11 \mathrm{~b}^{+}\right.$), dendritic cells (DCs, CD45 ${ }^{+} \mathrm{CD} 11 \mathrm{c}^{+}$ $\mathrm{MHC}$ II high), resident macrophage $\left(\mathrm{CD} 45^{+} \mathrm{CD} 11 \mathrm{~b}^{-}\right.$Siglec $\mathrm{F}^{+}$), eosinophils $\left(\mathrm{CD}_{4} 5^{+} \mathrm{CD} 11 \mathrm{~b}^{+}\right.$Siglec $\left.\mathrm{F}^{+}\right)$, monocyte-derived DCs or macrophages $\left(\mathrm{CD} 45^{+} \mathrm{Ly}_{6 \mathrm{C}^{+}} \mathrm{CD} 11 \mathrm{c}^{-}\right), \mathrm{CD} 4 \mathrm{~T}$ cells $\left(\mathrm{CD} 45^{+}\right.$ $\left.\mathrm{CD}^{+} \mathrm{CD}^{+}\right), \mathrm{CD} 8 \mathrm{~T}$ cells $\left(\mathrm{CD} 45^{+} \mathrm{CD}^{+} \mathrm{CD}^{+}\right)$, and $\mathrm{B}$ cells $\left(\mathrm{CD} 45^{+} \mathrm{CD} 19^{+}\right)$. DCs in lymph nodes were stained with extracellular CD45, CD11c, and CD80, and intracellular TNF $\alpha$, IFN $\gamma$, and IL-4. Total numbers of each cell population were calculated by multiplying the frequency of the population by the total number of leukocytes (the percentage of $\mathrm{CD} 45^{+}$cells multiplied by the original hemocytometer counts for total cells).

\section{Lung-Associated Lymph Node (LALN) Leukocyte Isolation}

Lung-associated lymph node leukocytes were collected as previously described with modifications (22). Lymph nodes were removed from the mediastinum and then mechanically dispersed using an 1-ml sterile syringe plunger to press them through a $70 \mu \mathrm{m}$ cell strainer (BD Falcon, Bedford, MA, USA) in complete medium. After centrifugation at 2,500 $\times g$ for $5 \mathrm{~min}$, the supernatant was removed and the cell pellets saved for further use.

\section{Immunoblot Analysis}

Lungs were ground up in liquid nitrogen and suspended in lysis buffer containing Tris- $\mathrm{HCl}(50 \mathrm{mM}$; $\mathrm{pH} 8.0), \mathrm{NaCl}(150 \mathrm{mM})$, EDTA (1 mM), NP-40 (1\%), PMSF (1 mM; Sigma), phosphatase inhibitor (Sigma), and a protease inhibitor cocktail (Roche Biochemical Laboratories). After incubation on ice for $30 \mathrm{~min}$, the cell lysates were collected after centrifugation $(14,000 \times g$ for $10 \mathrm{~min}$ ) at $4^{\circ} \mathrm{C}$, and protein concentrations were determined using the Pierce BCA Protein Assay Kit (Thermo Scientific). A total of $30 \mu \mathrm{g}$ protein was loaded for western blot analysis using the following antibodies: RIPK3 (Prosci) and caspase-3 (Cell Signaling Technology).

\section{Real-time PCR Analysis}

Total RNA (10-100 ng, depending on the abundance of the target gene) was purified using TRIzol reagent (Ambion by Life Technologies) for RT-qPCR in a one-step reaction with Reverse Transcriptase (Takara) and SYBR green master mix (Takara) using a 7900 Real-Time PCR system (Applied Biosystems). All primers used for RT-qPCR are listed in Table S1 in Supplementary Material. The qPCR analysis was performed using the $2^{-\Delta \mathrm{Ct}}$ method, and target genes were normalized to the housekeeping genes in each strain.

\section{Histology, Immunohistochemistry, and Immunofluorescence}

Lungs were instilled with $1 \mathrm{ml} 10 \%$ neutral-buffered formalin, excised, immersed in 10\% neutral-buffered formalin, and embedded in paraffin as described previously (19). Five-micrometer sections were cut and stained with hematoxylin \& eosin. Immunohistochemical and immunofluorescent staining was performed using formalin-fixed, paraffin-embedded tissue sections with rabbit anti-RIPK3 (Prosci) antibodies. Sections were photographed by Zeiss light microscopy (ZEISS, AXIO) and Olympus confocal microscopy (FV1000). Lung tissue inflammation and injury score were performed by three different pathologists in a blinded fashion. The quantify criteria is referenced to previous studies with modification (23).

\section{Fungal Killing and Cell Viability Assays}

Bone marrow-derived macrophages (BMDMs) were generated as previously described $(24,25)$. Briefly, marrow was flushed from the $\mathrm{C} 57 \mathrm{Bl} / 6$ mouse femurs and tibias and dispersed into a single-cell suspension. The cells were cultured for 7 days in RPMI medium supplemented with $10 \%$ FBS and $50 \mathrm{ng} / \mathrm{ml} \mathrm{M-CSF}$. The cultures were additionally nourished with M-CSF-containing 
medium on the third day of culture. All in vitro experiments were performed in RPMI 1640 containing 10\% FCS and 5 ng/ $\mathrm{ml}$ M-CSF.

For the fungal killing assay, freshly isolated BMDMs were diluted to $10^{6}$ cells $/ \mathrm{ml}$ and plated on a 96-well cell culture plate. C. neoformans were washed twice with PBS, resuspended in RPMI medium, and adjusted to $10^{5}$ cells $/ \mathrm{ml}$. The yeast cells were further opsonized with anti-GXM antibody for $1 \mathrm{~h}$ at $37^{\circ} \mathrm{C}$, followed by the addition of $100 \mu \mathrm{l}$ opsonized $C$. neoformans to each well of the $\mathrm{BMDM}$ culture plate and incubation at $37^{\circ} \mathrm{C}$ with $5 \% \mathrm{CO}_{2}$ for $24 \mathrm{~h}$. BMDM cells were lysed in sterile water for $20 \mathrm{~min}$, mixed with the supernatant, and then diluted and plated on Sabouraud agar plates. CFU were counted after 2 days at $30^{\circ} \mathrm{C}$.

\section{Statistical Analysis}

All data are expressed as means \pm SEMs. The data obtained for the animal survival assays were plotted as Kaplan-Meier survival curves and analyzed with the log-rank test using GraphPad Prism version 6.00 for Windows (GraphPad Software, San Diego, CA, USA). The remaining statistical analyses were conducted with the ANOVA, Student's $t$-test, and Kruskal-Wallis test as well as Dunn's test for non-parametric measures. The results were considered statistically significant when the $P$ value was less than 0.05 .

\section{RESULTS}

\section{RIPK3 and FADD Critically Contribute to Host Defenses against C. neoformans Infection}

To gain insight into the roles of RIPK3 and FADD during host responses to $C$. neoformans infection, we first evaluated kinetics of RIPK3 and FADD protein expression in the lungs of the infected C57BL6 mice. Immunoblot study results showed that RIPK3 displayed extensive upregulation in the lung tissue at 10 days postinfection (dpi) (Figure 1A), further verified by immunochemistry and immunofluorescence assays (Figures 1B,C). In contrast, FADD, albeit abundant, appeared to be expressed constitutively throughout the studied time points of infection. Next, to elucidate the relative importance of FADD and RIPK3-related signaling in anti-cryptococcal defense, we compared the survival of infected Ripk ${ }^{-1-}$, Ripk $3^{-1-} \mathrm{Fadd}^{-1-}$, and WT mice. As shown in Figure 1D, compared with WT mice, infected Ripk $3^{-1-}$ mice showed accelerated onset of mortality ( 8 vs $20 \mathrm{dpi}$ ) and reduced median survival (median: $16.5 \pm 5.8$ vs $21.5 \pm 1.4$ days). Ripk $3^{-/-}$Fadd $^{-1-}$ mice showed even greater susceptibility to $C$. neoformans infection compared with either Ripk $3^{-1-}(P<0.05)$ or WT mice $(P<0.001)$, exhibiting a drastically shortened median survival
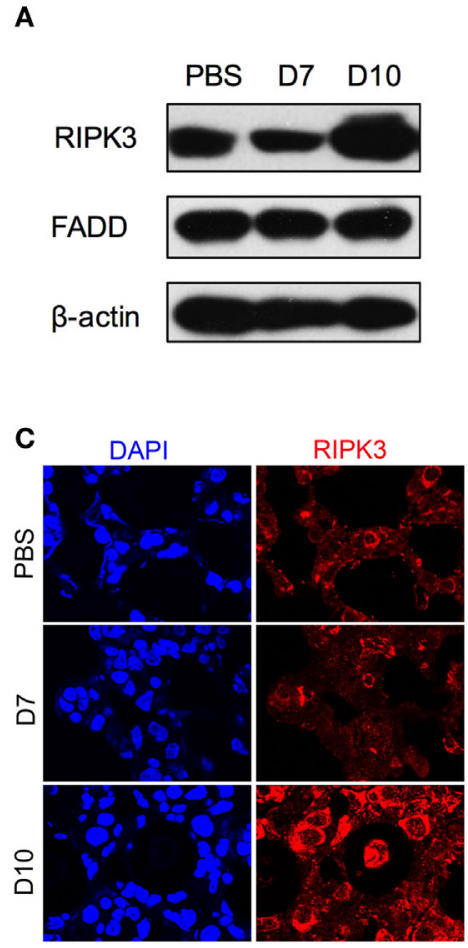

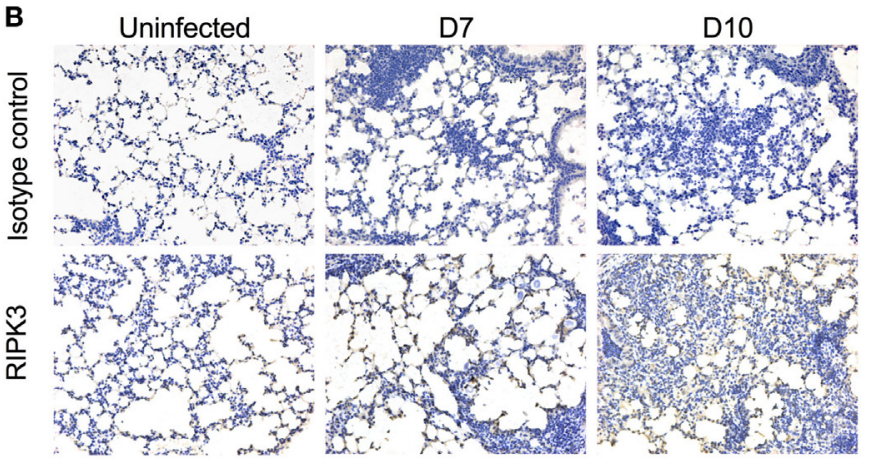

D

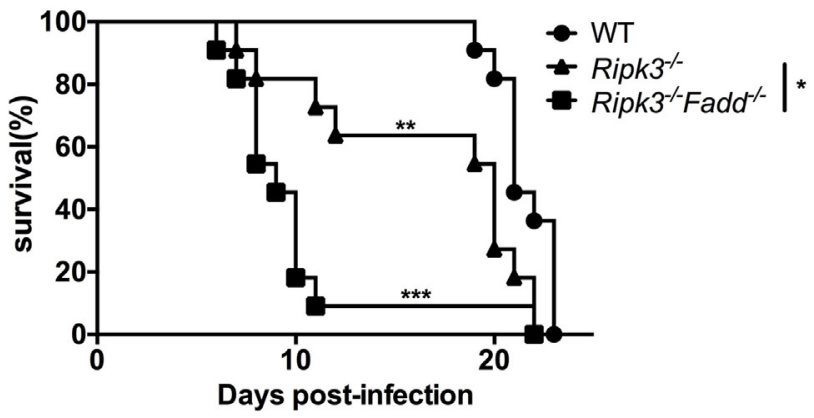

FIGURE 1 | RIPK3 and Fas-associated death domain (FADD) critically contribute to host defenses against Cryptococcus neoformans infection. C57BL/6 mice were inoculated intranasally with $10^{5} \mathrm{C}$. neoformans strain $\mathrm{H} 99$ and sacrificed at 7 or 10 days postinfection. PBS-treated mice were utilized as control. Pulmonary expression of RIPK3 and FADD were examined by western blot analysis in total protein form lung homogenates (A). RIPK3 displayed enhanced expression, while FADD remains unchanged in the lung of infected mice. Fungal infection significantly enhanced the local recruitment of RIPK3 in the lung as shown by

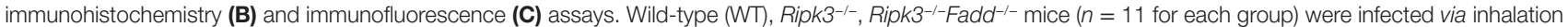

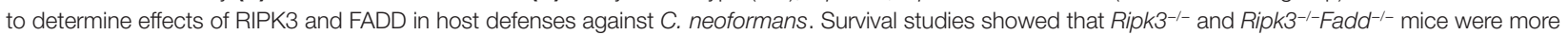
susceptible to fungal infection (D). ${ }^{\star} P<0.05,{ }^{\star \star} P<0.01,{ }^{\star \star \star} P<0.001$. The survival study was repeated three times independently. 
( $9.9 \pm 4.3$ days). These results demonstrate that FADD and RIPK3 signaling critically contribute to host defense against cryptococcal infection, most likely in a synergistic fashion.

\section{RIPK3 or RIPK3/FADD Deletion Reduced Host Ability to Control Fungal Growth and Dissemination}

Next, we examined fungal burden in the lung, brain, and spleen in infected mice to assess the effects of RIPK3 and FADD in the control of fungal growth and systemic dissemination. Compared with WT, Ripk $3^{-/-}$mice had a significantly higher pulmonary fungal load at $10 \mathrm{dpi}(P<0.05)$, and RIPK3/FADD double deletions further enhanced the fungal burden in the lungs at both $7 \mathrm{dpi}(P<0.05)$ and $10 \mathrm{dpi}$ (Figure 2A, $P<0.01)$. Similar trends in fungal burden were noted in the spleens and brains of Ripk $3^{-/}$ and $\mathrm{Ripk}^{-/-} \mathrm{Fadd}^{-/-}$mice at $10 \mathrm{dpi}$ (Figures 2B,C), with frequencies of positive spleen and brain cultures in $\mathrm{Ripk}^{-{ }^{-1}} \mathrm{Fadd}^{-{ }^{-}}$mice doubling those in the WT mice. Thus, both RIPK3 and FADD significantly contributed fungal containment during pulmonary cryptococcal infection.

\section{RIPK3/FADD Deletions Lead to Severe Lung Pathologies in C. neoformans-Infected Mice}

Rapid clinical deterioration and the accelerated mortality, especially in the infected Ripk $3^{-/} \mathrm{Fadd}^{-/-}$mice could not be solely explained by relatively modest increases in fungal burdens at 7 and 10 dpi. Thus, we examined the effects of RIPK3 or RIPK3/

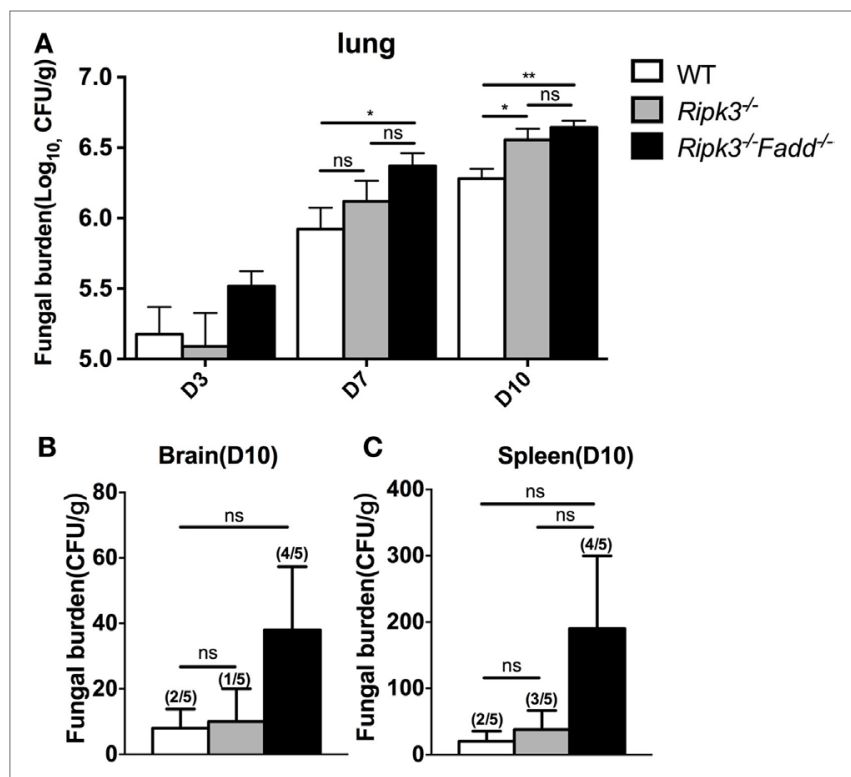

FIGURE 2 | Ripk3 $3^{-/-}$and Ripk3 $3^{-/-}$Fadd $^{-/-}$mice showed diminished fungal clearance. Pulmonary fungal burden assay revealed diminished fungal

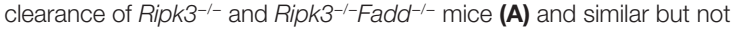
significant trends in extra-pulmonary dissemination (B,C). Values represent means and SEMs ( $n=5$ mice for each group); ns, no significant difference, ${ }^{\star} P<0.05,{ }^{* *} P<0.01$, between compared groups. The colony-forming units (CFU) assay was repeated three times independently.
FADD on the development of lung inflammation and pathologies post-C. neoformans infection. We examined lung sections obtained from sham-infected (PBS) and C. neoformans-infected WT and Ripk $3^{-/-}$or Ripk $3^{-/}$Fadd $^{-/-}$mice. Deletion of RIPK3 or RIPK3/FADD induced no visible alterations in uninfected lungs (data not shown), demonstrating that genetic defects in RIPK3 or RIPK3/FADD did not affect baseline pulmonary morphology. Comparative histopathological assessments of lung sections from each group at $10 \mathrm{dpi}$ (Figure 3 ) demonstrated only subtle enhancement of pulmonary leukocyte infiltration in $R i p \mathrm{k}^{-/-}$mice relative to the control mice with largely similar pattern of inflammatory lesions (Figures 3A-C vs Figures 3D-F). The borders between inflamed regions and normal alveoli remained distinct at $10 \mathrm{dpi}$ in WT and $\mathrm{Ripk}^{-/-}$mice (Figures 3A-F). Consistently, blinded pathology score, albeit showing an increasing trend, has not increased significantly (Figure S2 in Supplementary Material). In contrast, Ripk $3^{-I-} \mathrm{Fadd}^{-1-}$ mice exhibited progressive pulmonary inflammation with severe tissue damage (Figures 3G-I). Less organized inflammatory infiltrates (predominantly neutrophils and lymphocytes) were spread diffusely through bilateral lung fields at $10 \mathrm{dpi}$. The margins of inflamed regions from uninvolved alveoli were less distinct. Features of suppurative bronchopneumonia, such as airway plugins with polymorphic neutrophil and dense cellular exudate, were observed throughout the lung (Figures 3H,I). The blinded pathology score showed that inflammation/pathology score was significantly greater in lungs of Ripk3 $^{-I-} \mathrm{Fadd}^{-1-}$ mice compared to both WT and Ripk $3^{-1-}$ mice (Figure S2 in Supplementary Material), which corroborated well with survival data on day 10 (80\% mortality in Ripk $3^{-1-} \mathrm{Fadd}^{-1-}$ group, only $20 \%$ mortality in Ripk $3^{-/}$group, and all mice surviving in the WT group at $10 \mathrm{dpi})$. Collectively, these findings demonstrated that concurrent RIPK3/FADD deletion during C. neoformans infection induced severe pulmonary inflammation and tissue damage, potentially explaining the highly accelerated

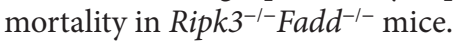

\section{RIPK3 and FADD Differentially Modulated Pulmonary Leukocyte Accumulation during $C$. neoformans Infection}

To quantify the effects of RIPK3 and FADD deletions on cellular components of the inflammatory response to C. neoformans, we compared leukocyte populations isolated from uninfected or infected lungs of WT, Ripk $3^{-/}$, or Ripk ${ }^{-/-} \mathrm{Fadd}^{-/}$mice at $10 \mathrm{dpi}$. Consistent with the histopathological findings, flow cytometric analysis revealed no significant effect of Ripk $3^{-/-}$or Ripk $3^{-1-} \mathrm{Fadd}^{-1-}$ mutations in uninfected mice for any of the leukocyte subsets (data not shown). While only a borderline increase in total lung leukocytes counts $\left(\mathrm{CD} 45^{+}\right.$cells) was observed in $\mathrm{Ripk}^{-/-}$mice compared to WT mice at 10 dpi (Figure 4A, $P<0.06$ ), significant increases in two pulmonary leukocyte subsets were observed. Ripk $3^{-/-}$mice showed increased numbers of neutrophils (Figure 4B), increasing trend in macrophages (Figure 4C), and elevated $\mathrm{CD}^{+} \mathrm{T}$ cells (Figure 4D) compared to the infected WT mice.

Ripk $3^{-1-} \mathrm{Fadd}^{-1-}$ mice infected by C. neoformans showed more profound alterations in both the magnitude of inflammation 

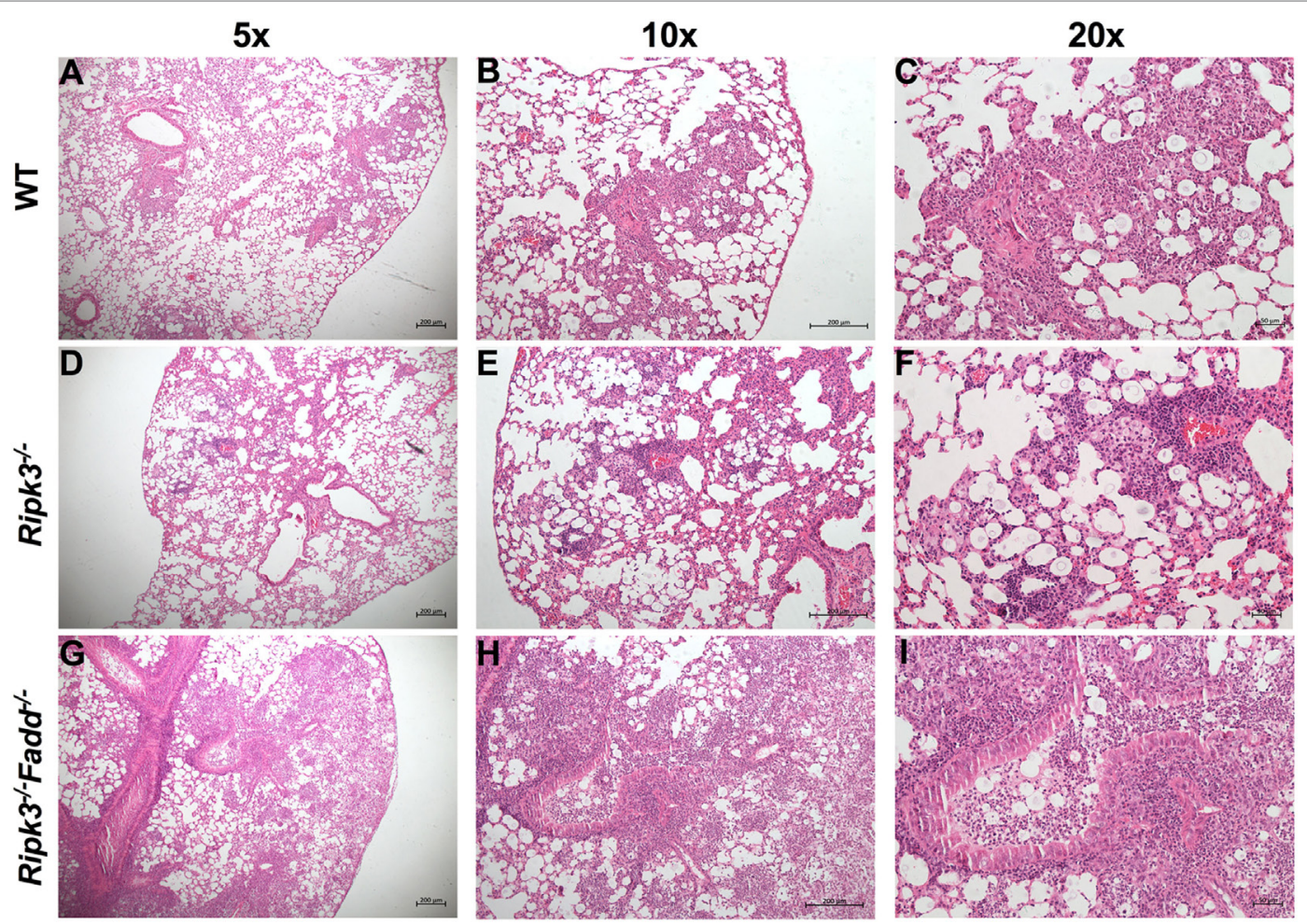

FIGURE 3 | Deletion of RIPK3 and Fas-associated death domain (FADD) resulted in enhanced inflammatory infiltration and tissue injury following pulmonary infection

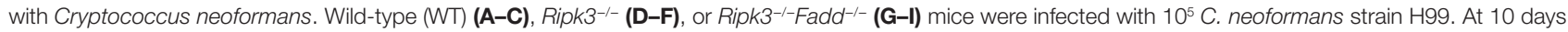
postinfection, lungs were harvested, and the morphological pattern of inflammation was evaluated by light microscopy. Pulmonary representative photomicrographs (hematoxylin and eosin stained) taken from each group were shown [(A,D,G) magnification of $\times 50 ; \mathbf{( B , E , H ) ~ m a g n i f i c a t i o n ~ o f ~} \times 100$; $(\mathbf{C}, \mathbf{F}, \mathbf{I})$ magnification of $\times 200]$. Compared with WT group, Ripk3-/- mice displayed similar pulmonary histological pattern but a slight enhancement in inflammatory infiltration. However, extensive

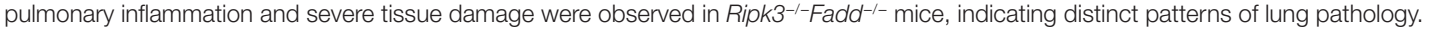

and leukocyte composition. There was a significant increase in total leukocyte counts in $\mathrm{Ripk}^{-/-} \mathrm{Fadd}^{-/-}$mice compared with WT group at $10 \mathrm{dpi}$ (Figure 4A), mostly driven by a remarkable increase in neutrophil numbers (more than threefold) relative to infected WT (Figure 4B) and significantly greater than in the Ripk $3^{-1-}$ mice. Significant increases in other myeloid cell subsets (monocytes, Figure 4E; DCs, Figure 4F) were also detected relative to both WT and Ripk $3^{-1-}$ mice. However, the numbers of pulmonary eosinophils observed in abundance in the WT and

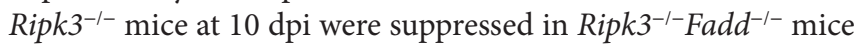
(Figure 4G), suggesting a shift away from a Th2 response in the WT following RIPK3/FADD double deletion. Collectively, these data further support that RIPK3 and more so the RIPK3/ FADD double deletion results in an exuberant accumulation of inflammatory cells and immunomodulation that alters the course of inflammatory response implicated in lung injury we observe during C. neoformans infection in the absence of these factors.

\section{RIPK3/FADD Deletion Reshaped the Cytokine Responses during Pulmonary C. neoformans Infection}

Having demonstrated that RIPK3 and RIPK3/FADD deletions altered inflammatory infiltrate compositions, we further examined the roles of RIPK3 and FADD in pulmonary and systemic cytokine levels during cryptococcal infection. Lung homogenates and serum isolated at $10 \mathrm{dpi}$ from Ripk $^{-/-}, \mathrm{Ripk}^{-/-}$ $\mathrm{Fadd}^{-1-}$, and WT mice were analyzed by ELISA (Figures 5A-N). We found that depletion of RIPK3 alone significantly increased pro-inflammatory cytokine production: TNF- $\alpha$, IL- $1 \alpha$, and IL-1 $\beta$ (Figures 5A-C). Non-significant increasing trend in IFN- $\gamma$ level and decreasing trend in IL- 4 production suggested cytokine profile drifting away from Th2 to Th1 pattern (Figure 5J), which corresponded to diminished serum IgE accumulation (Figure 5N). However, no difference in IL-12p40 or IL-33 have been observed (Figures $\mathbf{5 E}, \mathbf{H}$ ), suggesting that the full switch from Th2 to Th1 has not occurred as a result of RIPK3 deletion. Finally, while we observe significant increase in IL-17A, which might have suggested shift toward Th17 response as a result of RIPK3 deletion, there was no concurrent increase in IL-6 or IL-12p40, which would be associated with Th17 response. Most of these trends were reproduced in pulmonary leukocyte cell culture supernatants (Figure S4 in Supplementary Material). Consistently, with lung cytokines, serum cytokine analysis showed somewhat elevated $\mathrm{TNF} \alpha$ (Figure 5K), suggesting more pronounced inflammatory response levels in infected Ripk $3^{-/-}$mice, but no increase in serum IL-6 or IFN $\gamma$ (Figures 5L,M). 

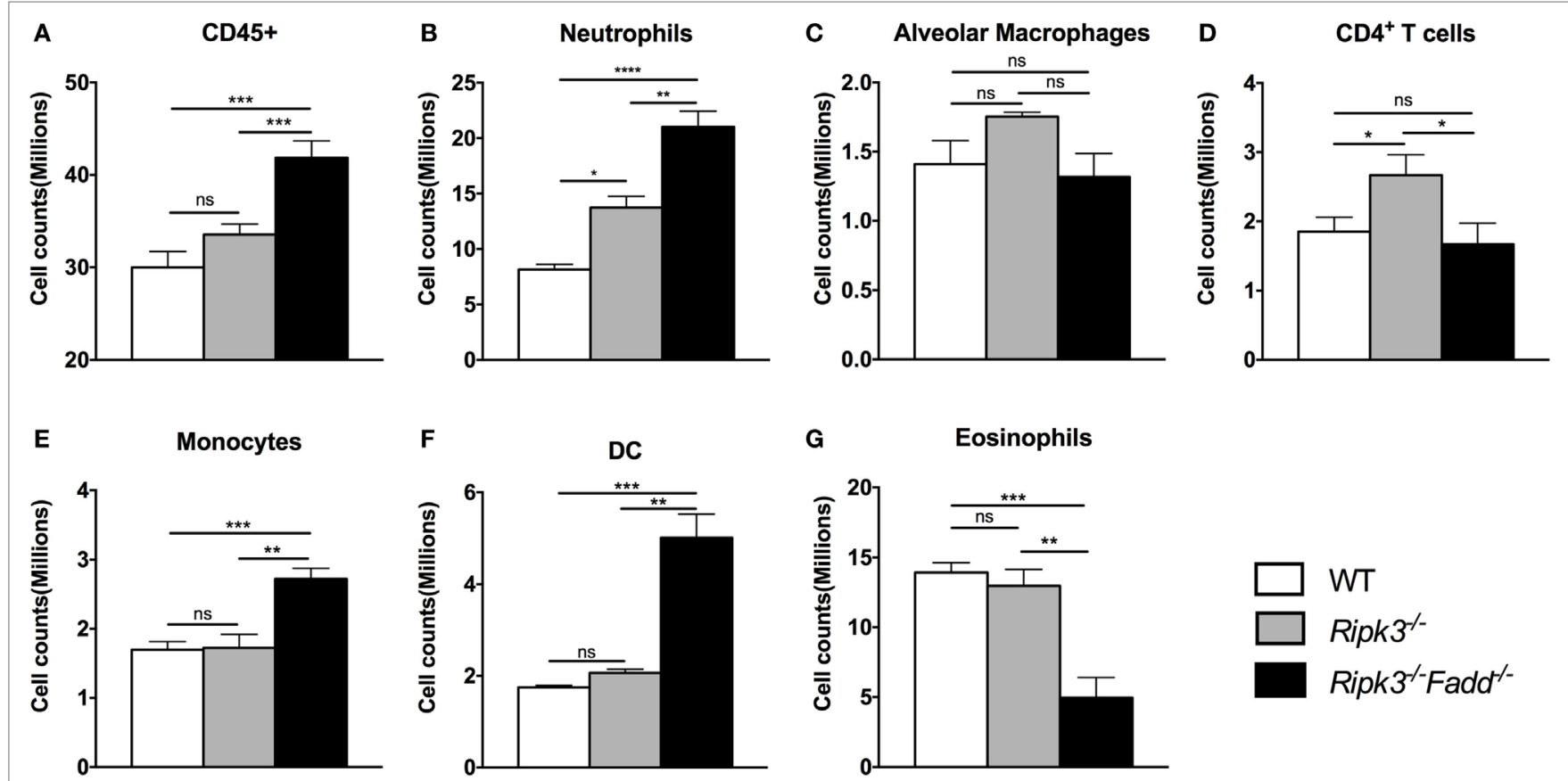

FIGURE 4 | RIPK3 or RIPK3/Fas-associated death domain (FADD) deletions resulted in alteration in pulmonary leukocyte recruitments. Mice were inhalationally infected with $10^{5}$ Cryptococcus neoformans strain H99. Lungs were harvested at 10 days postinfection, and total pulmonary leukocytes were isolated from infected lungs after enzymatic digestion. Magnitude and/or leukocyte composition were then analyzed by flow cytometry. Results are illustrated as absolute numbers of each lymphocyte subset (A-G) in the total lung sample. Values represent means and SEMs ( $n=6$ mice per group). ns, no significant difference, ${ }^{\star} P<0.05$, ${ }^{\star \star} P<0.01$, ${ }^{\star \star \star} P<0.001,{ }^{\star \star \star \star} P<0.0001$, between compared groups. This experiment was repeated twice.

RIPK3/FADD double deletion further enhanced proinflammatory TNF $\alpha$ and IL-6 (Figures 5A,D) and showed sustained elevation of IL- $1 \alpha$, IL- $1 \beta$ compared to the WT mice. Furthermore, Th1 cytokines IL-12p40 and IFN $\gamma$ were significantly elevated relative to WT and RIPK3 mice levels of these cytokines (Figures 5E,F), suggesting not only strongly intensified inflammatory response but also shift to a Th1. Consistently, Th2 cytokines (IL-4 and IL-33) were profoundly suppressed (Figures 5G,H), and the Th1/Th2 ratio increased more than 300fold in RIPK3/FADD-depleted relative to WT mice (Figure 5J), with corresponding absence of serum $\operatorname{IgE}$ accumulation (Figure 5N) strongly suggesting the development of a robust Th1 bias in these mice. Interestingly, IL-17 was not elevated as in the Ripk $3^{-1-}$ mice but showed level similar to that in the infected WT mice (Figure 5I).

To further investigate effects of RIPK3/FADD on T cell polarization, we performed intracellular flow analysis on pulmonary $\mathrm{CD}^{+} \mathrm{T}$ cells from infected $\mathrm{Ripk}^{-1-}$ and $\mathrm{Ripk}^{{ }^{-1-}} \mathrm{Fadd}^{-1-}$ mice at $10 \mathrm{dpi}$. Consistent with the cytokine data, percentage of IFN $\gamma^{+}$ $\mathrm{CD} 4^{+} \mathrm{T}$ cells was not different in Ripk $3^{-1-}$ mice compared to the WT, but increased in Ripk $3^{-/} \mathrm{Fadd}^{-/-}$mice compared to WT and Ripk $3^{-1-}$ mice (Figures 6A,B). Moreover, we found there was a borderline reduction in the frequencies of GATA3 positive $\mathrm{CD} 4^{+}$ $\mathrm{T}$ cells in Ripk ${ }^{--}(P=0.07)$ mice and significant decrease in frequencies of $\mathrm{GATA}^{+} \mathrm{CD}^{+} \mathrm{T}$ cells in $\mathrm{Ripk3}^{-1-} \mathrm{Fadd}^{-1-}$ mice relative to $\mathrm{WT}$ mice at $10 \mathrm{dpi}$ (Figures 6C,D). Interestingly, no increase in IL-17A or Ror $\gamma \mathrm{T}^{+} \mathrm{CD} 4$ or CD8 T-cells was observed in

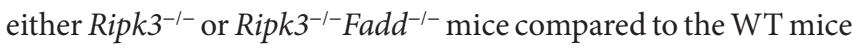
(data not shown). Collectively, analysis of cytokine responses and T-cell polarization profile showed that RIPK3 single deletion led to enhanced pro-inflammatory responses, while that additional deletion of FADD further potentiated these effects, leading to a very strong Th1 bias systemic and "cytokine storm" at $10 \mathrm{dpi}$ with C. neoformans.

\section{RIPK3 and FADD Deletions Potentiated Classical Activation of Macrophages and Their Fungicidal Responses In Vitro}

Macrophages are distal effector cells that execute antiC. neoformans-based cytokine responses in infected organs $(26,27)$. Having determined that RIPK3 increased inflammatory cytokines, including pro-M1 cytokine TNF $\alpha$ and RIPK3/ FADD additionally promoted Th1 responses, we assessed the M1/M2 polarization patterns of macrophages in the infected lungs and the fungicidal ability of BMDMs isolated from uninfected mice from each strain. The expression of M1- and M2-associated genes was evaluated by real-time PCR (Figures 7A-C). Consistent with absence of major increase in IFN $\gamma$ production, Ripk $3^{-1-}$ mice macrophages did not show significant upregulation of the M1 activation marker iNOS (Figure 7A). However, in concert with less pronounced Th2 and more pro-inflammatory environment in the lungs, we observed diminished upregulation of M2 markers arginase Arg1 and Fizz1 


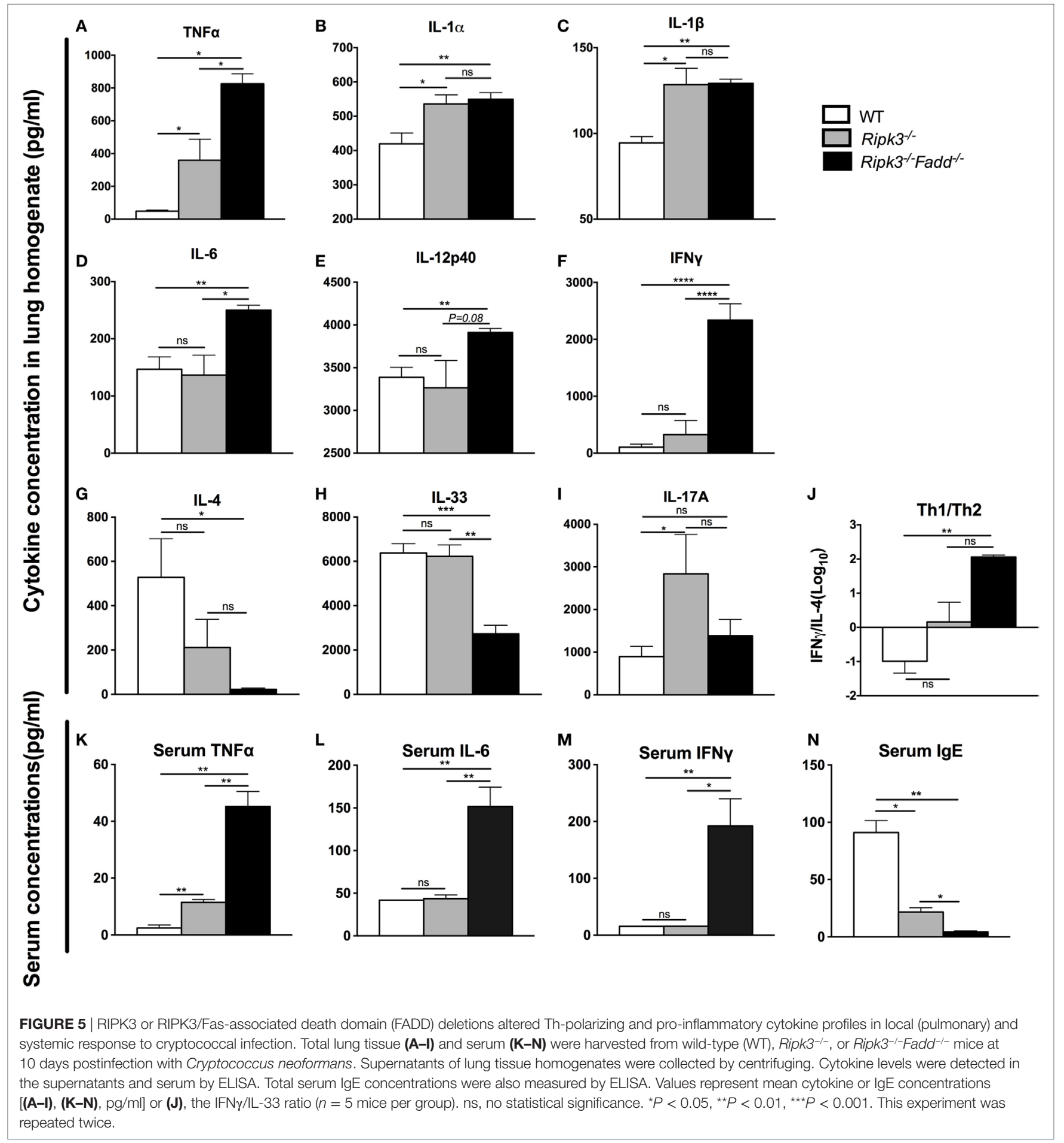

(Figures 7B,C), suggesting that macrophages in the infected lung of Ripk $3^{--}$mice were less M2 biased. Consistent with the strong Th1-type polarization, expression of the iNOS was significantly upregulated in $\mathrm{Ripk3}^{-l-} \mathrm{Fadd}^{-/-}$mice (Figure 7A), while Fizzl expression was diminished (Figure 7C) compared to both WT and Ripk3 ${ }^{-l-}$ mice. Compared with WT mice, arginase 1 expression in Ripk3 $^{-/-} \mathrm{Fadd}^{-/-}$mice also showed downward effects (Figures 7B,C). To determine whether the fungicidal potential (typically linked to M1/M2 activation) was affected in Ripk $^{-1-}$ and $\mathrm{Ripk}^{-1-} \mathrm{Fadd}^{-1-}$ mice, we evaluated the fungicidal effect of the BMDMs after co-incubation with $10^{6} \mathrm{CFU}$ of C. neoformans (Figure 7D). Significantly reduced cryptococcal survival was observed in $R i p k 3^{-/-}$and, to an even greater extent, in Ripk3 ${ }^{-/-}$Fadd $^{-1-}$ macrophages, further demonstrating 

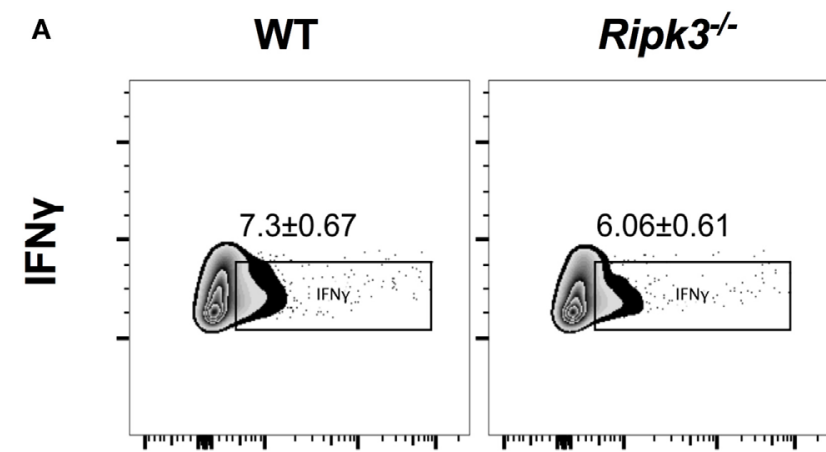

Ripk3-'-Fadd ${ }^{-1-}$

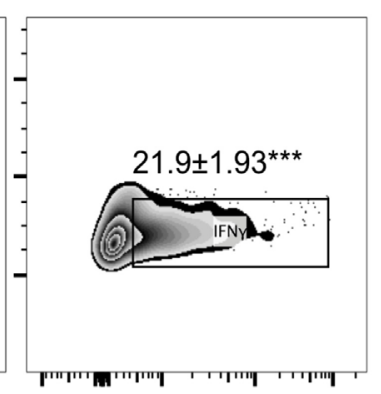

C
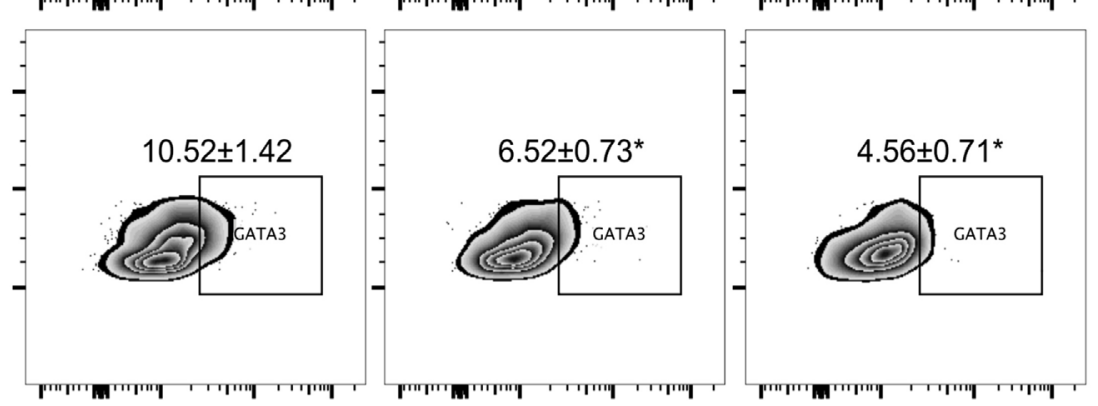

B

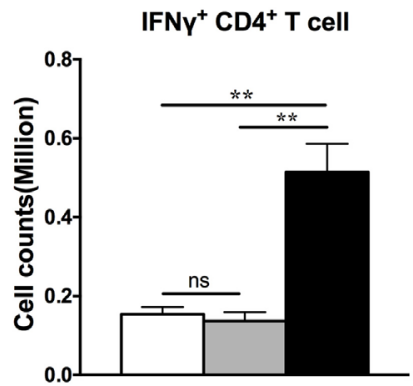

D

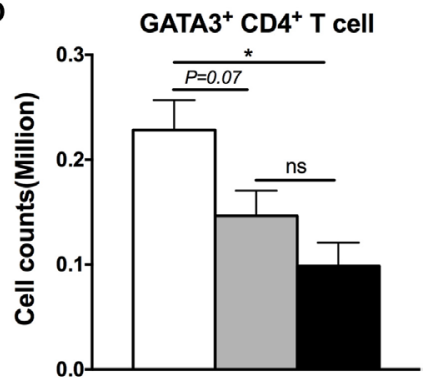

FIGURE 6 | RIPK3 or RIPK3/Fas-associated death domain (FADD) deletions affects Th1/Th2 polarization of CD4 T cells in the lungs of Cryptococcus neoformansinfected mice. Leukocytes were isolated from the lungs of infected mice on 9 days postinfection. Intracellular expression of IFN $\gamma$, GATA3 in CD4 T cell population was analyzed by flow cytometry. (A,C) show the percentage of IFN $\gamma$ or GATA positive populations in CD4+ cells. (B,D) show the cell counts of IFN $\gamma$ or GATA positive

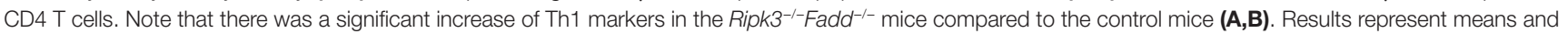
SEMs ( $n=4$ mice for each time point). ${ }^{\star} P<0.05,{ }^{\star \star} P<0.01$.
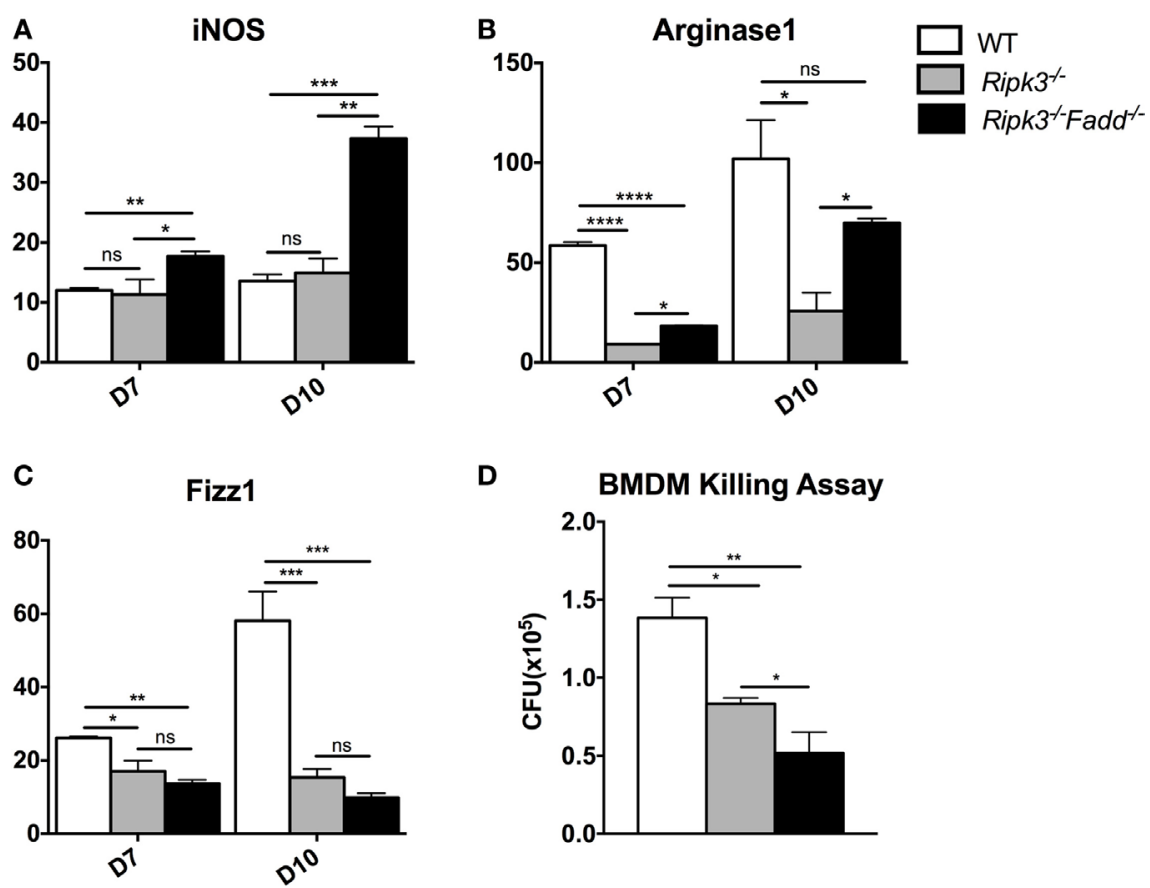

D

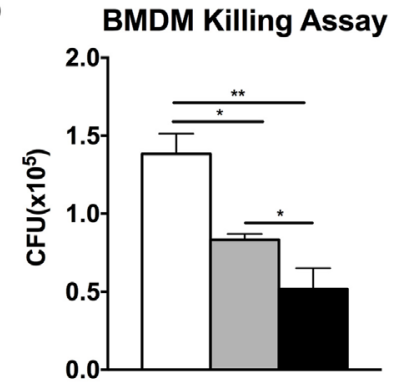

FIGURE 7 | Ripk3-/- and Ripk3 ${ }^{-/-}$Fadd $^{-/-}$mice displayed progressively classical activation and increased fungicidal ability of macrophages. mRNA was isolated from

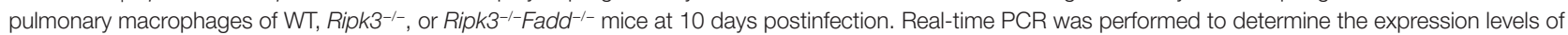
iNOS [nitric oxide synthase, (A)], Arg1 [arginase 1, (B)], and Fizz1 (C). Bone marrow-derived macrophages (BMDMs) isolated from uninfected mice were utilized to detect intracellular fungicidal ability (D). ns, no significant difference, ${ }^{\star} P<0.05,{ }^{\star \star} P<0.01,{ }^{\star \star \star} P<0.001,{ }^{\star \star \star \star} P<0.0001$, between compared groups. These experiments were repeated three times. 
that, at the cellular level, RIPK3 and FADD deletions promoted rather than suppressed fungicidal M1 macrophage polarization. Thus, the effects of RIPK3 and FADD deletion on M1/M2 gene expression were consistent with the cytokine profiles in the C. neoformans-infected lungs.

\section{RIPK3 and FADD Deletions Promoted DC Differentiation and Activation in LALNs after Cryptococcal Infection}

Having determined that joint RIPK3/FADD deletion promoted a strong shift toward the Th1/M1 response in contrast with single RIPK3 deletion, we analyzed the phenotype of DCs, the central regulators of Th-immune polarization. Flow cytometric analysis of LALN DC was performed to examine the frequency and intensity of CD80, a major co-stimulatory molecule expressed during DC co-stimulatory maturation, and the DC1 phenotypic marker and predictor of Th1 response development $(22,28,29)$. Consistent with strong Th1 bias, the frequency and intensity of CD80 expression was significantly enhanced in LALN DC of Ripk $^{-1-} \mathrm{Fadd}^{-1-}$ mice at both 7 and 10 dpi (Figures 8A-D) compared to those in the infected WT mice. Ripk $3^{-/-}$mice also did not show this effect on day 7 but displayed some increase of CD80 surface expression at $10 \mathrm{dpi}$ (Figures 8C,D). Cytokine expression in LALN DCs was also analyzed by intracellular staining. Again, notable enhancement of IFN $\gamma$ expression was observed in LALN DCs of $\mathrm{Ripk}^{-1-} \mathrm{Fadd}^{-1-}$ mice at day 7 but without significant alteration in IL-4 production (Figures 8E,F). However, RIPK3 deletion had no effect on LALN DC cytokine expression. Taken together, these results suggested that RIPK3/FADD deletions synergistically promoted LALN DC maturation and DC1 activation during $C$. neoformans infection, which was consistent with strongly enhanced Th1 responses in these mice, while changes in Ripk $3^{-l-}$ DC activation profile was subtle, consistent with subtle
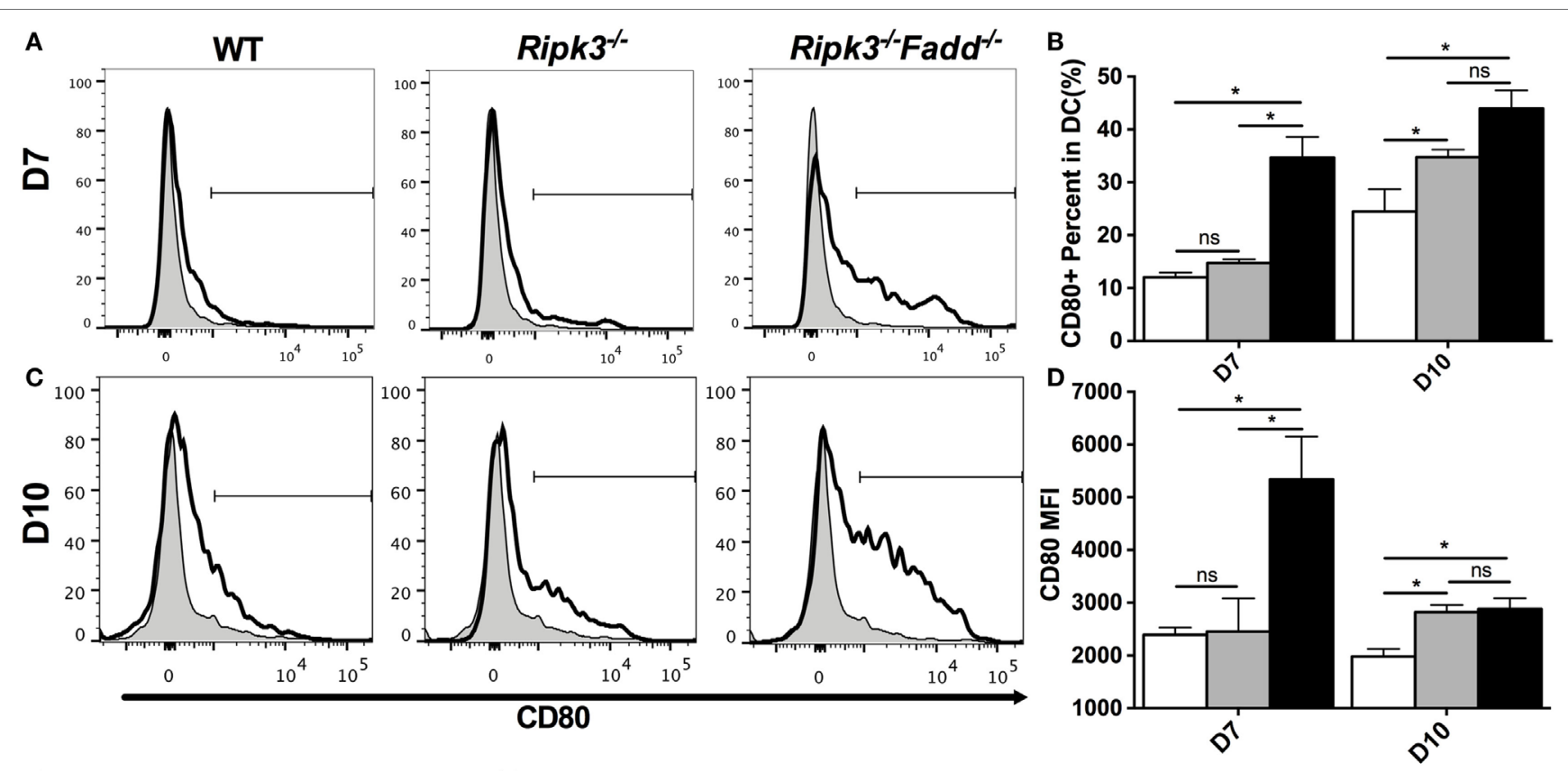

E

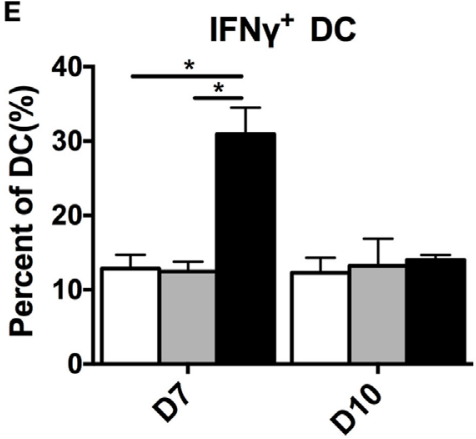

$\mathbf{F}$

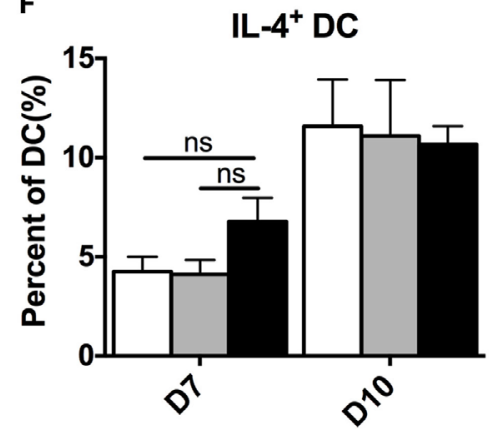

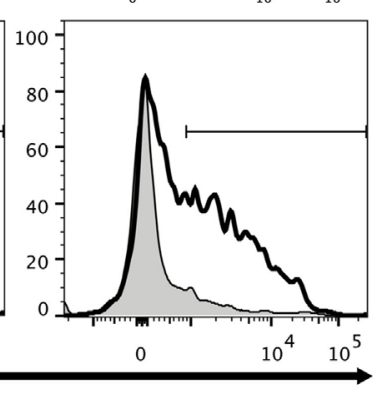

IL-4+ DC

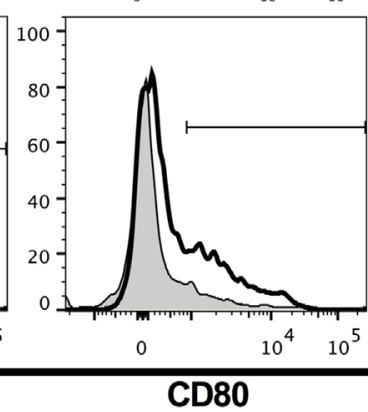

$10^{4} \quad 10^{5}$

FIGURE 8 | RIPK3 and Fas-associated death domain (FADD) deletion promoted dendritic cells differentiation and activation in lung-associated lymph nodes (LALNs)

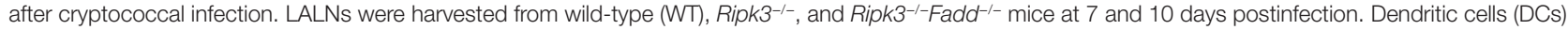
were stained with CD80 (A-D), IFN $\mathbf{( E )}$, and IL-4 (F) antibodies and then analyzed by flow cytometry. Data are expressed as means and SEMs $(n=3$ or more mice per group). ns, no significant difference, ${ }^{*} P<0.05$, between compared groups. This experiment was repeated twice. 
effects of Ripk $^{-/-}$Th polarization profile during C. neoformans infection.

\section{RIPK3 and FADD Deletions Differentially Affected Apoptosis Rate in Leukocyte Subsets during $C$. neoformans Infection}

To further explore the potential mechanisms underlying how RIPK3 and RIPK3/FADD pathways prevent excessive accumulation of inflammatory cells, we next evaluated the apoptotic frequency (APF) of various pulmonary cell subsets in $C$. neoformans-infected lungs. Lungs were dissociated gently, and single-cell suspensions were analyzed by flow cytometry with annexin V. Since different leukocyte subsets have distinct life spans and exploit different cell death pathways $(30,31)$, we first tested their APF in each group without fungal infection to exclude direct effects of FADD and/or RIPK3 deletions. All three groups exhibited similar rates of apoptosis in all subsets, indicating that FADD and RIPK3 were dispensable for homeostatic survival of these immune cells under physiological condition (data not shown).

Similar APFs in each cell subset at 10 dpi in Ripk3 $3^{-1-}$ compared with WT mouse infected lungs (Figure 9 and Figure S4 in Supplementary Material) suggested that RIPK3 alone had no effect on inflammatory cell apoptosis during $C$. neoformans infection. However, C. neoformans infection significantly reduced eosinophil cell death in WT and Ripk $3^{-1-}$ mice (eosinophil APF from uninfected mice: $60.9 \% \pm 1.4 \%$ decreasing to $6.6 \pm 1.2 \%$

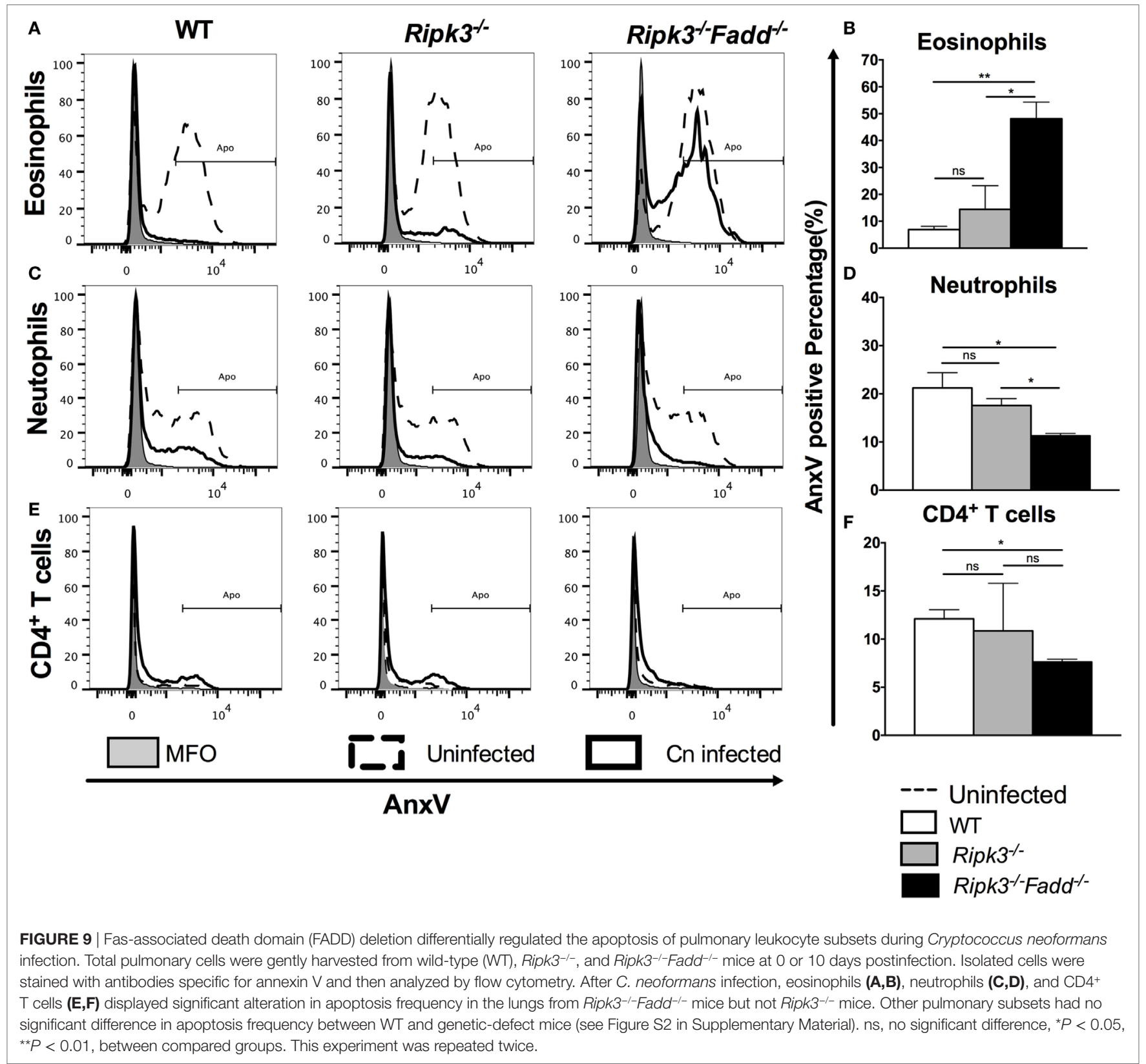


and $16.3 \pm 8.0$, respectively), whereas the absence of FADD completely abolished this phenomenon (Figures 9A,B). A considerable reduction of neutrophil cell death was also detected in each group after fungal infection. Furthermore, consistent with the greatest increase in neutrophil accumulation, the Ripk $3^{-/} \mathrm{Fadd}^{-/-}$ group exhibited significant decrease in the APF of neutrophils $(11.0 \% \pm 0.9 \%)$ compared with WT $(21.5 \pm 3.2 \%, P<0.05)$ or Ripk ${ }^{-1-}$ mice (17.6 $\pm 1.5 \%$, Figures 9C,D). Among lymphocyte subsets, only $\mathrm{CD}^{+} \mathrm{T}$ cells displayed a significant reduction in cell death frequency in $\mathrm{Ripk}^{-/-} \mathrm{Fadd}^{-/-}$mice $(12.1 \pm 1.7$ vs $7.6 \pm 0.5 \%, P<0.05$ ), while other subpopulations did not differ in each group (Figures 9E,F). Collectively, these data indicated that $C$. neoformans infection affected apoptotic rates of certain leukocyte subsets, but only the joint FADD/RIPK3 deletion led to detectable alterations in the APF in these leukocyte subsets during fungal infection.

\section{The Effects of RIPK3 and FADD Deletions on Anti-Cryptococcal Defenses Appear to Be Unrelated to Their Role Necroptosis Pathway}

RIPK3 and FADD, among other functions, are major intracellular upstream mediators of necroptosis cell death pathway, a PCD resulting in cell lysis, reported to influence mycobacterial-infected macrophages (32) but also to regulate type-I IFN signaling in macrophages independent of necroptosis in influenza-infected macrophages (33). We first asked a question about the global role of necroptosis pathway in anti-cryptococcal host defenses. To asses this, mice with deletion of a distal effector kinase in necroptosis pathway, MLKL along with the WT mice, were infected with C. neoformans and mouse survival was monitored. Results show that MLKL deletion had no significant effect on C. neoformansinfected mouse survival (Figure 10A) demonstrating that the necroptosis cell death pathway is dispensable for mouse resistance to $C$. neoformans.

We next asked, whether C. neoformans infection triggers significant level of lytic cell death in macrophages and whether this was affected by RIPK3 and FADD deletion. To address this question, we used LDH release assay on WT, Ripk $3^{-/-}$, Ripk $3^{-/-} \mathrm{Fadd}^{-/-}$ BMDM following in vitro H99-challenge. Results show that $C$. neoformans infection triggered lytic death in a small subset of $\operatorname{BMDM}(14.3 \pm 1.7 \%$, Figure 10B), The cell death rate was potentiated when the cells were treated with $C$. neoformans opsonized with M18B7 antibody, which increases cryptococcal uptake by macrophages (34) reaching $(21.7 \pm 1.6 \%$, Figure 10B). However, neither RIPK3 deletion alone nor combined with FADD deletion had effect on the rate of BMDM death. This was in contrast with the positive control, in which lytic cell death triggered by the combined simulation of lipopolysaccharide with pan-caspase inhibition was profoundly reduced Ripk3 ${ }^{-1-}$, Ripk3 ${ }^{-1-} \mathrm{Fadd}^{-1-} \mathrm{BMDM}$ (Figure 10B). Thus, RIPK3 and FADD are not directly involved in regulation lytic cell death of BMDM population infected with $C$. neoformans in vitro, further supporting that the susceptibility of Ripk $3^{-1-}$ mice and Ripk $3^{-1-} \mathrm{Fadd}^{-1-}$ mice to cryptococcal infection was mechanistically unrelated to the role RIPK3 and FADD in necroptosis pathway.
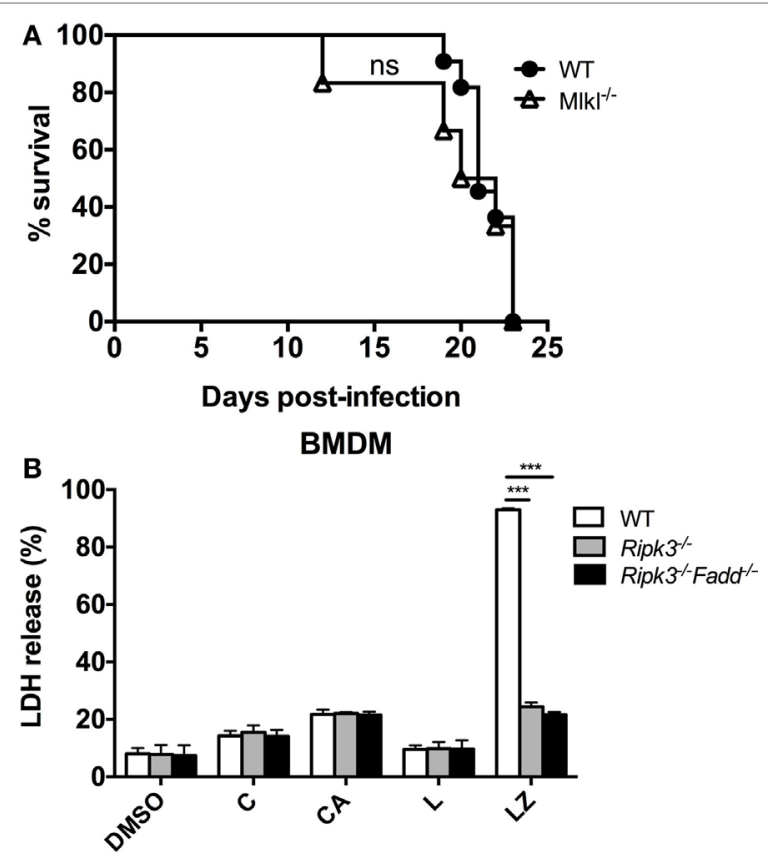

FIGURE 10 | Necroptosis process is not likely a mechanism for RIPK3- and Fas-associated death domain (FADD)-mediated effects on immune responses to Cryptococcus neoformans. (A) Wild-type (WT) mice and mice with targeted deletion of MLKL gene (distal effector kinase in necroptosis pathway) were infected with $C$. neoformans and analyzed as in Figure 1D. $M L K L$ deletion had no effects on survival, unlike RIPK3 and FADD deletions (per Figure 1D) ( $n=10$ for each group). (B) Bone marrow-derived macrophage (BMDM) cells isolated form WT, Ripk3 ${ }^{-/-}$, Ripk3 ${ }^{-/-}$Fadd $^{-/-}$mice were challenged with $\mathrm{H} 99$ at $\mathrm{MOI}=5: 1$ and co-incubated for $24 \mathrm{~h}$. Supernatants were collected followed by $\mathrm{LDH}$ release detection. In RIPK3 or FADD, deletions had no effect on magnitude of BMDM lytic death in contrast with their effects in positive control $(L Z)$. Study was repeated three times independently. Symbols signify C, C. neoformans; A, anti-cryptococcal antibody M18B7; Z, zVAD (pan-caspase inhibitor); L, lipopolysaccharide; ns, no significant difference, ${ }^{\star \star \star} P<0.001$, between compared groups.

\section{DISCUSSION}

While PCD pathway components were shown to be important regulators of the immune responses, the role of FADD and RIPK3 in host defenses against fungal infection remained unknown up to this point. This report provides novel data demonstrating that RIPK3 and FADD are crucial for fungal containment and survival of the infected host during C. neoformans infection. Here, we show that these factors serve jointly as physiological "brake" that prevents the development of over exuberant inflammation and profound Th1 bias, which in their absence leads to pulmonary damage and rapid deterioration of the infected host.

Our first group of studies elucidated the involvement of both RIPK3 and FADD during cryptococcal infection. Upon C. neoformans infection, RIPK3 expression in infected lungs was strongly upregulated and deletion of RIPK3 significantly shortened the survival of the infected mice and impaired fungal clearance. Furthermore, the absence of RIPK3 resulted in upregulation of pro-inflammatory components including, increased $\mathrm{T}$ cell numbers and neutrophils and increased inflammatory 
cytokines in response to C. neoformans (Figures $\mathbf{5}$ and 6). These data provide evidence that RIPK3 exerts an important role in controlling inflammatory responses with some minor effect on Th polarization during C. neoformans infection that is at least in part independent of its well-established function in apoptosis and necroptosis pathways (Figures 9 and 10).

While FADD expression appeared to be constitutive, RIPK3/ FADD double deletion resulted in further enhanced susceptibility to C. neoformans infection, demonstrating that joint absence of these factors further potentiated the effects of single RIPK3 deletion at both severity of pathology and upregulation of inflammatory components in the infected lungs. In Ripk $3^{-/} \mathrm{Fadd}^{-/-}$ mice, lung pathology and the effect on mouse survival effects were more severe (Figures 1-3; Figure S2 in Supplementary

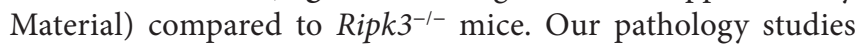
further highlighted that in C. neoformans-infected lungs RIPK3 and FADD play important roles in protecting infected lungs against the rapid development of severe pathology associated with excessive accumulation and activation of leukocytes. One caveat here could be that the enhanced inflammatory pathology was driven by the increased fungal burden; however, the lung CFU burdens even in the most profoundly affected Ripk $3^{--I} \mathrm{Fadd}^{-/-}$mice were still relatively modest and could not explain the profound pathological changes and $80 \%$ mortality at day 10 in these mice. This, together with very selective amplification of Th1 response, clearly indicates that rather small differences in fungal burdens were not the major driver of the profound changes in the immunophenotype and increase in mouse mortality.

Inflammation is a double-edged sword in the pathogenesis of infectious disease. While suboptimal production of pro-inflammatory cytokines hinders control of $C$. neoformans infection (35-37), highly elevated production of these factors promotes severe inflammatory tissue damage (38). In at least some aspects of host-fungus interactions, a preservation immune strategy denoted "protective tolerance" may be optimal to limit immunopathology while controlling fungal infection (39). This is the first report that RIPK3 and FADD may play jointly important roles by critically fine-tuning "protective tolerance" mechanisms during anti-cryptococcal defense. We show that both of these two molecules are required for optimal control of fungal growth and host protection against the severe inflammatory pathology that develops in lungs in the absence of these factors. Interestingly, the phenotypes that developed in Ripk $3^{-/} \mathrm{Fadd}^{-/}$mice mirror many findings in cryptococcosis patients who suffer from IRIS. The development of IRIS (pre-IRIS phase) is specifically characterized by increasing pro-inflammatory responses without efficient clearance of the fungal pathogen $(40,41)$. Several cohort studies highlighted the relationship of IL- 6 signaling and the risk of developing IRIS. For every twofold increase in IL-6 or C-reactive protein, the hazard of IRIS increased by 1.6 and 1.5 , respectively (42). Another recent study proposed TNF $\alpha$, IL-1 $\beta$, and IL-12 to be predictors of IRIS (43), and these factors found to be elevated as a result of RIPK3 and FADD deletion in C. neoformansinfected lungs. Finally, a central IRIS characteristic reproduced in $\mathrm{Ripk3}^{-/-} \mathrm{Fadd}^{-/-}$mice is massive systemic elevation of IFN $\gamma$, TNF $\alpha$, and IL-6 (42) (Figure 5).
Besides the findings that mimic cytokine profiles of IRIS patients in our models, we also found the elevated induction of IL-1 $\alpha$ and neutrophil recruitment in the lungs of both Ripk3 $^{-/-}$and Ripk3 ${ }^{-/} \mathrm{Fadd}^{-/-}$mice. Neutrophil recruitment linked to IL-1 $\alpha$ upregulation have been shown to contribute to the development of lethal lung pathology during fungal infection with Aspergillus (44). Together, these findings document that responses "designed" to be protective in fungal infections can become highly detrimental to the host, and our data demonstrate such detrimental outcomes following RIPK3 and FADD deletion. Collectively, our study shows that RIPK3 and FADD factors are crucial elements of regulatory network that allows protecting the host from pathological effects of inflammation while supporting clearance of the invasive fungal infection.

Another novel finding was that the expression of RIPK3 and FADD proteins was required for the development of Th2 polarization during $C$. neoformans infection. While the deletion of RIPK3 had subtle effect on Th2 polarization, joint deletion of RIPK3 and FADD resulted in a complete switch from strong Th2 to strong Th1 response. Cytokine profile analysis revealed massive reduction in Th2 cytokines (IL-4 and IL-33) in infected lungs of Ripk3 ${ }^{-1-} \mathrm{Fadd}^{-/-}$mice. This, together with diminished GATA3 expression by $\mathrm{CD} 4 \mathrm{~T}$ cells and the absence of serum IgE accumulation (Figures 5 and 6), indicates the loss of Th2 polarization in response to RIPK 3 and FADD deletion. Th1 and Th2 responses are known to counterbalance each other. Therefore, it remains to be determined if the primary effect of RIPK3/FADD deletion was the lack of the Th2 development with its subsequent "replacement by Th1," other way around, or else both pathways were subjected to concurrent regulation by joint action of RIPK3 and FADD. While future studies are needed to address these points, our data show that the net effect of RIPK3 and FADD is directly or indirectly counter-regulate Th1 and support Th2 polarization during C. neoformans infection. Another highly unexpected finding was that strong $\mathrm{Th} 1$ bias and the absence of Th 2 rather than being protective $(19,45)$ resulted in non-protective response in the infected Ripk $3^{-1-} \mathrm{Fadd}^{-1-}$ mice. This absence of improved clearance following RIPK3/FADD deletions, despite the robust Th1 polarization, was not due to a defect in macrophage M1 polarization or loss of their intrinsic fungicidal function downstream of robust M1 polarization. Our studies of macrophages from infected lungs and BMDMs revealed neither a defect in macrophage polarization nor a defect in their effector (killing) functions resulting from RIPK3 or FADD deletion (Figure 7). In spite of this, we observed paradoxical impairment of pulmonary fungal clearance in both Ripk3 $^{--}$and Ripk3 $3^{-1-} \mathrm{Fadd}^{-1-}$ mice (Figure 2). One explanation for this observation is that the excessive systemic activation of microbicidal factors in macrophages resulted in exhaustion of these effectors cells even before they reached the infection site, which seems to be consistent with our data (Figures 4 and 7). Additionally, the excessive accumulation of neutrophils at the infection site interfered with macrophage and T-cell fungicidal functions, since an excessive accumulation of neutrophils have been reported to contribute to tissue damage and defects in the clearance of other fungal organisms $(46,47)$.

The final observation in our study is quite strong independence of immunoregulatory effects exerted by RIPK3 and FADD 
from apoptotic or necroptotic cell death. The diminished apoptosis likely contributed to the excessive accumulation of neutrophils in infected Ripk $3^{-l} \mathrm{Fadd}^{-/-}$mice (Figures 3, 4B and 9D) since, granulocytes are typically eliminated via extrinsic apoptosis after a brief period of activation (48). However, the enhanced neutrophil accumulation was also observed in Ripk $3^{-1-}$ mice without significant effect on neutrophil APF. Likewise, APF in eosinophil population was greatest in the infected Ripk $3^{-1-} \mathrm{Fadd}^{-/}$mice, demonstrating that eosinophil apoptosis did not require or was positively regulated by FADD or RIPK3. Furthermore, RIPK3 and FADD deletion appeared not to affect APF in T and B cells, monocytes, DCs, or epithelial cells in the infected lungs. Finally, our outcomes do not favor necroptosis as an important pathway defining host-pathogen interactions during C. neoformans infection (Figure 10). Thus, while future studies are needed to provide definitive answers, our data favor the hypothesis that RIPK 3 and FADD can induce immunoregulatory effects during fungal infection in a manner independent of their role in PCD responses, but chiefly by regulating cytokine responses.

In summary, our results demonstrate, for the first time, that RIPK 3 and FADD are vital components of the immune responses to fungal pathogens. These molecules are required for fine-tuning of inflammatory responses during infection, acting as powerful regulators of Th1 and Th2 polarization. They contribute to optimal fungal clearance and serve as indispensable "nodes" in immunoregulatory network supporting tissue damage control in fungal-infected host.

\section{ETHICS STATEMENT}

This study was carried out in accordance with the recommendations of National Institutes of Health Guide for the Care and
Use of Laboratory Animals. The protocol was approved by the Scientific Investigation Board of Second Military Medical University.

\section{AUTHOR CONTRIBUTIONS}

ZF, QX, and WF contributed to study concept and design, performing the experiments, and drafting of the manuscript. $\mathrm{WL}, \mathrm{MO}, \mathrm{XD}$, and $\mathrm{HZ}$ (Haibing Zhang) contributed to study concept and design, analysis, and interpretation of data, critical revision of the manuscript, obtained funding, and provided study supervision, administrative, and technical support. HZ (Haiwei Zhang), JX (Jintao Xu), WP, and JX (Jinhua Xu) contributed to data analysis and revision of the manuscript. All listed authors gave final approval of the manuscript.

\section{FUNDING}

This study was supported by the National Key Basic Research Programs of China (2013CB531601 and 2013CB531606), the National Natural Science Foundation of China (81401651, 81471926, 81271799, and 81501728), China Postdoctoral Science Foundation Grant (2016M600286), and Shanghai Key Laboratory of Molecular Medical Mycology (14DZ2272900). MO's work is supported by Merit Review Grant 1I01BX000656 and VA Research Career Scientist Award 1K6BX003615 from the US Department of Veterans' Affairs.

\section{SUPPLEMENTARY MATERIAL}

The Supplementary Material for this article can be found online at http://journal.frontiersin.org/article/10.3389/fimmu. 2017.01055/full\#supplementary-material.

\section{REFERENCES}

1. Fang W, Fa Z, Liao W. Epidemiology of Cryptococcus and cryptococcosis in China. Fungal Genet Biol (2015) 78:7-15. doi:10.1016/j.fgb.2014.10.017

2. Heitman J, Kozel TR, Kwon-Chung KJ, Perfect JR, Casadevall A. Cryptococcus: From Human Pathogen to Model Yeast. Washington, DC: ASM Press (2010). 275 p.

3. Maziarz EK, Perfect JR. Cryptococcosis. Infect Dis Clin North Am (2016) 30(1):179-206. doi:10.1016/j.idc.2015.10.006

4. Greenlee-Wacker MC. Clearance of apoptotic neutrophils and resolution of inflammation. Immunol Rev (2016) 273(1):357-70. doi:10.1111/imr.12453

5. Ortega-Gomez A, Perretti M, Soehnlein O. Resolution of inflammation: an integrated view. EMBO Mol Med (2013) 5(5):661-74. doi:10.1002/emmm. 201202382

6. Scaffidi C, Volkland J, Blomberg I, Hoffmann I, Krammer PH, Peter ME. Phosphorylation of FADD/MORT1 at serine 194 and association with a 70-kDa cell cycle-regulated protein kinase. J Immunol (2000) 164(3):1236-42. doi:10.4049/jimmunol.164.3.1236

7. Zhang J, Kabra NH, Cado D, Kang C, Winoto A. FADD-deficient T cells exhibit a disaccord in regulation of the cell cycle machinery. JBiol Chem (2001) 276(32):29815-8. doi:10.1074/jbc.M103838200

8. Balachandran S, Thomas E, Barber GN. A FADD-dependent innate immune mechanism in mammalian cells. Nature (2004) 432(7015):401-5. doi:10.1038/ nature 03124

9. Ma Y, Liu H, Tu-Rapp H, Thiesen HJ, Ibrahim SM, Cole SM, et al. Fas ligation on macrophages enhances IL-1R1-toll-like receptor 4 signaling and promotes chronic inflammation. Nat Immunol (2004) 5(4):380-7. doi:10.1038/ni1054

10. Zhang J, Cado D, Chen A, Kabra NH, Winoto A. Fas-mediated apoptosis and activation-induced T-cell proliferation are defective in mice lacking FADD/ Mort1. Nature (1998) 392(6673):296-300. doi:10.1038/32681

11. Varfolomeev EE, Schuchmann M, Luria V, Chiannilkulchai N, Beckmann JS, Mett IL, et al. Targeted disruption of the mouse caspase 8 gene ablates cell death induction by the TNF receptors, Fas/Apol, and DR3 and is lethal prenatally. Immunity (1998) 9(2):267-76. doi:10.1016/S1074-7613(00) 80609-3

12. He S, Wang L, Miao L, Wang T, Du F, Zhao L, et al. Receptor interacting protein kinase-3 determines cellular necrotic response to TNF-alpha. Cell (2009) 137(6):1100-11. doi:10.1016/j.cell.2009.05.021

13. Kasof GM, Prosser JC, Liu D, Lorenzi MV, Gomes BC. The RIP-like kinase, RIP3, induces apoptosis and NF-kappaB nuclear translocation and localizes to mitochondria. FEBS Lett (2000) 473(3):285-91. doi:10.1016/ S0014-5793(00)01473-3

14. Moriwaki K, Balaji S, McQuade T, Malhotra N, Kang J, Chan FK. The necroptosis adaptor RIPK3 promotes injury-induced cytokine expression and tissue repair. Immunity (2014) 41(4):567-78. doi:10.1016/j.immuni. 2014.09.016

15. Shlomovitz I, Zargrian S, Gerlic M. Mechanisms of RIPK3-induced inflammation. Immunol Cell Biol (2017) 95(2):166-72. doi:10.1038/icb. 2016.124

16. Zhang X, Fan C, Zhang H, Zhao Q, Liu Y, Xu C, et al. MLKL and FADD are critical for suppressing progressive lymphoproliferative disease and activating the NLRP3 inflammasome. Cell Rep (2016) 16(12):3247-59. doi:10.1016/j. celrep.2016.06.103 
17. Semighini CP, Averette AF, Perfect JR, Heitman J. Deletion of Cryptococcus neoformans AIF ortholog promotes chromosome aneuploidy and fluconazole-resistance in a metacaspase-independent manner. PLoS Pathog (2011) 7(11):e1002364. doi:10.1371/journal.ppat.1002364

18. Meng Y, Zhang C, Yi J, Zhou Z, Fa Z, Zhao J, et al. Deubiquitinase Ubp5 is required for the growth and pathogenicity of Cryptococcus gattii. PLoS One (2016) 11(4):e0153219. doi:10.1371/journal.pone.0153219

19. Zhang Y, Wang F, Tompkins KC, McNamara A, Jain AV, Moore BB, et al. Robust Th1 and Th17 immunity supports pulmonary clearance but cannot prevent systemic dissemination of highly virulent Cryptococcus neoformans H99. Am J Pathol (2009) 175(6):2489-500. doi:10.2353/ajpath.2009.090530

20. Qiu Y, Dayrit JK, Davis MJ, Carolan JF, Osterholzer JJ, Curtis JL, et al. Scavenger receptor A modulates the immune response to pulmonary Cryptococcus neoformans infection. J Immunol (2013) 191(1):238-48. doi:10.4049/jimmunol. 1203435

21. Stevens WW, Kim TS, Pujanauski LM, Hao X, Braciale TJ. Detection and quantitation of eosinophils in the murine respiratory tract by flow cytometry. JImmunol Methods (2007) 327(1-2):63-74. doi:10.1016/j.jim.2007. 07.011

22. Xu J, Eastman AJ, Flaczyk A, Neal LM, Zhao G, Carolan J, et al. Disruption of early tumor necrosis factor alpha signaling prevents classical activation of dendritic cells in lung-associated lymph nodes and development of protective immunity against cryptococcal infection. MBio (2016) 7(4):e00510-16. doi:10.1128/mBio.00510-16

23. Szarka RJ, Wang N, Gordon L, Nation PN, Smith RH. A murine model of pulmonary damage induced by lipopolysaccharide via intranasal instillation. J Immunol Methods (1997) 202(1):49-57. doi:10.1016/S0022-1759(96)00236-0

24. Davis MJ, Tsang TM, Qiu Y, Dayrit JK, Freij JB, Huffnagle GB, et al. Macrophage M1/M2 polarization dynamically adapts to changes in cytokine microenvironments in Cryptococcus neoformans infection. MBio (2013) 4(3):e00264-13. doi:10.1128/mBio.00264-13

25. Davis MJ, Eastman AJ, Qiu Y, Gregorka B, Kozel TR, Osterholzer JJ, et al. Cryptococcus neoformans-induced macrophage lysosome damage crucially contributes to fungal virulence. J Immunol (2015) 194(5):2219-31. doi:10.4049/jimmunol.1402376

26. Alanio A, Desnos-Ollivier M, Dromer F. Dynamics of Cryptococcus neoformans-macrophage interactions reveal that fungal background influences outcome during cryptococcal meningoencephalitis in humans. MBio (2011) 2(4):e00158-11. doi:10.1128/mBio.00158-11

27. Arora S, Olszewski MA, Tsang TM, McDonald RA, Toews GB, Huffnagle GB. Effect of cytokine interplay on macrophage polarization during chronic pulmonary infection with Cryptococcus neoformans. Infect Immun (2011) 79(5):1915-26. doi:10.1128/IAI.01270-10

28. Herring AC, Falkowski NR, Chen GH, McDonald RA, Toews GB, Huffnagle GB. Transient neutralization of tumor necrosis factor alpha can produce a chronic fungal infection in an immunocompetent host: potential role of immature dendritic cells. Infect Immun (2005) 73(1):39-49. doi:10.1128/ IAI.73.1.39-49.2005

29. Eastman AJ, Osterholzer JJ, Olszewski MA. Role of dendritic cell-pathogen interactions in the immune response to pulmonary cryptococcal infection. Future Microbiol (2015) 10(11):1837-57. doi:10.2217/fmb.15.92

30. Hampson P, Hazeldine J, Lord JM. Neutrophil apoptosis and its induction as a potential treatment for chronic inflammatory disease. Curr Opin Hematol (2013) 20(1):10-5. doi:10.1097/MOH.0b013e32835b06be

31. Shen ZJ, Malter JS. Determinants of eosinophil survival and apoptotic cell death. Apoptosis (2015) 20(2):224-34. doi:10.1007/s10495-014-1072-2

32. Zhao X, Khan N, Gan H, Tzelepis F, Nishimura T, Park SY, et al. Bcl-xL mediates RIPK3-dependent necrosis in M. tuberculosis-infected macrophages. Mucosal Immunol (2017). doi:10.1038/mi.2017.12

33. Downey J, Pernet E, Coulombe F, Allard B, Meunier I, Jaworska J, et al. RIPK3 interacts with MAVS to regulate type I IFN-mediated immunity to influenza A virus infection. PLoS Pathog (2017) 13(4):e1006326. doi:10.1371/journal. ppat. 1006326
34. Zebedee SL, Koduri RK, Mukherjee J, Mukherjee S, Lee S, Sauer DF, et al. Mouse-human immunoglobulin G1 chimeric antibodies with activities against Cryptococcus neoformans. Antimicrob Agents Chemother (1994) 38(7):1507-14. doi:10.1128/AAC.38.7.1507

35. Bauman SK, Huffnagle GB, Murphy JW. Effects of tumor necrosis factor alpha on dendritic cell accumulation in lymph nodes draining the immunization site and the impact on the anticryptococcal cell-mediated immune response. Infect Immun (2003) 71(1):68-74. doi:10.1128/IAI.71.1.68-74.2003

36. Siddiqui AA, Shattock RJ, Harrison TS. Role of capsule and interleukin-6 in long-term immune control of Cryptococcus neoformans infection by specifically activated human peripheral blood mononuclear cells. Infect Immun (2006) 74(9):5302-10. doi:10.1128/IAI.00661-06

37. Axton PJ, Bancroft GJ. In vivo analysis of immune responses to Cryptococcus neoformans - role of interferon-gamma in host resistance. Biochem Soc Trans (1997) 25(2):276S. doi:10.1042/bst025276s

38. Osterholzer JJ, Milam JE, Chen GH, Toews GB, Huffnagle GB, Olszewski MA. Role of dendritic cells and alveolar macrophages in regulating early host defense against pulmonary infection with Cryptococcus neoformans. Infect Immun (2009) 77(9):3749-58. doi:10.1128/IAI.00454-09

39. Roussey JA, Olszewski MA, Osterholzer JJ. Immunoregulation in fungal diseases. Microorganisms (2016) 4(4):E47. doi:10.3390/microorganisms4040047

40. Skiest DJ, Hester LJ, Hardy RD. Cryptococcal immune reconstitution inflammatory syndrome: report of four cases in three patients and review of the literature. J Infect (2005) 51(5):e289-97. doi:10.1016/j.jinf.2005.02.031

41. Wiesner DL, Boulware DR. Cryptococcus-related immune reconstitution inflammatory syndrome (IRIS): pathogenesis and its clinical implications. Curr Fungal Infect Rep (2011) 5(4):252-61. doi:10.1007/s12281-011-0064-8

42. Boulware DR, Meya DB, Bergemann TL, Wiesner DL, Rhein J, Musubire A, et al. Clinical features and serum biomarkers in HIV immune reconstitution inflammatory syndrome after cryptococcal meningitis: a prospective cohort study. PLoS Med (2010) 7(12):e1000384. doi:10.1371/journal.pmed.1000384

43. Sun J, Chen H, Xie Y, Su J, Huang Y, Xu L, et al. Nuclear factor of activated T cells and cytokines gene expression of the $\mathrm{T}$ cells in AIDS patients with immune reconstitution inflammatory syndrome during highly active antiretroviral therapy. Mediators Inflamm (2017) 2017:1754741. doi:10.1155/2017/1754741

44. Caffrey AK, Lehmann MM, Zickovich JM, Espinosa V, Shepardson KM, Watschke CP, et al. IL-1alpha signaling is critical for leukocyte recruitment after pulmonary Aspergillus fumigatus challenge. PLoS Pathog (2015) 11(1): e1004625. doi:10.1371/journal.ppat.1004625

45. Holmer SM, Evans KS, Asfaw YG, Saini D, Schell WA, Ledford JG, et al. Impact of surfactant protein D, interleukin-5, and eosinophilia on cryptococcosis. Infect Immun (2014) 82(2):683-93. doi:10.1128/IAI.00855-13

46. Zelante T, De Luca A, D'Angelo C, Moretti S, Romani L. IL-17/Th17 in anti-fungal immunity: what's new? Eur J Immunol (2009) 39(3):645-8. doi:10.1002/eji.200839102

47. Zelante T, De Luca A, Bonifazi P, Montagnoli C, Bozza S, Moretti S, et al. IL-23 and the Th17 pathway promote inflammation and impair antifungal immune resistance. Eur J Immunol (2007) 37(10):2695-706. doi:10.1002/eji.200737409

48. Fox S, Leitch AE, Duffin R, Haslett C, Rossi AG. Neutrophil apoptosis: relevance to the innate immune response and inflammatory disease. J Innate Immun (2010) 2(3):216-27. doi:10.1159/000284367

Conflict of Interest Statement: The authors declare that the research was conducted in the absence of any commercial or financial relationships that could be construed as a potential conflict of interest.

Copyright (® 2017 Fa, Xie, Fang, Zhang, Zhang, Xu, Pan, Xu, Olszewski, Deng and Liao. This is an open-access article distributed under the terms of the Creative Commons Attribution License (CC BY). The use, distribution or reproduction in other forums is permitted, provided the original author(s) or licensor are credited and that the original publication in this journal is cited, in accordance with accepted academic practice. No use, distribution or reproduction is permitted which does not comply with these terms. 